\title{
Time Course of Forward Masking Tuning Curves in Cat Primary Auditory Cortex
}

\author{
MICHAEL BROSCH AND CHRISTOPH E. SCHREINER \\ W. M. Keck Center for Integrative Neuroscience, University of California at San Francisco, San Francisco, \\ California 94143-0732
}

Brosch, Michael and Christoph E. Schreiner. Time course of forward masking tuning curves in cat primary auditory cortex. J. Neurophysiol. 77: 923-943, 1997. Nonsimultaneous two-tone interactions were studied in the primary auditory cortex of anesthetized cats. Poststimulatory effects of pure tone bursts (masker) on the evoked activity of a fixed tone burst (probe) were investigated. The temporal interval from masker onset to probe onset (stimulus onset asynchrony), masker frequency, and intensity were parametrically varied. For all of the 53 single units and 58 multiple-unit clusters, the neural activity of the probe signal was either inhibited, facilitated, and/or delayed by a limited set of masker stimuli. The stimulus range from which forward inhibition of the probe was induced typically was centered at and had approximately the size of the neuron's excitatory receptive field. This "masking tuning curve" was usually V shaped, i.e., the frequency range of inhibiting masker stimuli increased with the masker intensity. Forward inhibition was induced at the shortest stimulus onset asynchrony between masker and probe. With longer stimulus onset asynchronies, the frequency range of inhibiting maskers gradually became smaller. Recovery from forward inhibition occurred first at the lower- and higher-frequency borders of the masking tuning curve and lasted the longest for frequencies close to the neuron's characteristic frequency. The maximal duration of forward inhibition was measured as the longest period over which reduction of probe responses was observed. It was in the range of $53-430 \mathrm{~ms}$, with an average of $143 \pm 71$ (SD) ms. Amount, duration and type of forward inhibition were weakly but significantly correlated with "static" neural receptive field properties like characteristic frequency, bandwidth, and latency. For the majority of neurons, the minimal inhibitory masker intensity increased when the stimulus onset asynchrony became longer. In most cases the highest masker intensities induced the longest forward inhibition. A significant number of neurons, however, exhibited longest periods of inhibition after maskers of intermediate intensity. The results show that the ability of cortical cells to respond with an excitatory activity depends on the temporal stimulus context. Neurons can follow higher repetition rates of stimulus sequences when successive stimuli differ in their spectral content. The differential sensitivity to temporal sound sequences within the receptive field of cortical cells as well as across different cells could contribute to the neural processing of temporally structured stimuli like speech and animal vocalizations.

\section{INTRODUCTION}

Many natural sounds and communication sounds consist of temporal sequences of spectrally complex acoustic events. In speech, important information-carrying temporal features are the duration, separation, and order of individual segments. Depending on their spectral composition, duration, and temporal separation, successive auditory events may be perceived as a single auditory stream or are segregated into different auditory streams (Bregman 1990). Numerous psychophysical studies have demonstrated that the temporal stimulus context affects the perceptual quality of individual auditory events. Detection thresholds of individual sounds can be elevated (Lüscher and Zwislocki 1947), and the subjective pitch and loudness (Stevens and Davis 1938) of auditory events can be altered by preceding and succeeding sounds. Although the perceptual consequences of the temporal stimulus context have been studied intensively, little information is available on the central neural mechanisms and neural structures underlying the processing of time-varying stimuli.

Among the different neural structures, the auditory cortex has been recognized as playing an important role in the processing of temporal stimulus sequences. The main experimental support for this comes from behavior-lesion studies on cats and monkeys. After ablation of large parts of the temporal lobe, subjects are significantly impaired in discriminating changes in the temporal patterns of tone sequences (Diamond and Neff 1957; Diamond et al. 1962; Kaas et al. 1967; Strominger et al. 1980) and tone durations (Scharlock et al. 1965). Lesions also result in permanent incapacities in discrimination of species-specific vocalizations in squirrel monkeys (Hupfer et al. 1977) and in macaques (Heffner and Heffner 1984), and induce aphasia-like deficits (Heffner and Heffner 1989).

The cortical encoding of time-varying stimuli has mainly been analyzed with simple, uniformly composed stimulus sequences. Recordings of neural discharges in auditory cortex have demonstrated that cells usually respond transiently to the onset of sounds. The capacity of neurons to represent time-varying signals is, however, limited. With the use of stimulus sequences of the pure tones or clicks, a number of studies have shown that neurons respond in a time-locked fashion to repetition rates of up to several elements per second (Creutzfeldt et al. 1980; Eggermont 1991; Phillips et al. 1989; but see also de Ribaupierre et al. 1972). At higher repetition rates neurons typically only respond to the first element of the sequence but not at all or only weakly to the following elements. Similar results were obtained in studies with periodically modulated sounds (Eggermont 1994; Schreiner and Langner 1988; Schreiner and Urbas 1986, 1988). Neurons at subcortical stages of the auditory pathway, in contrast, generally respond to much higher repetition rates (e.g., Langner 1992). From these results it has been suggested that cortical neurons code for the time structure of transients of auditory signals, as first suggested by Creutz- 
feldt et al. (1980). There is anecdotal evidence that cells in auditory cortex might also serve as sequence detectors (McKenna et al. 1989; Riquimaroux 1994). Responses to individual pure tones could be enhanced or attenuated if they were succeeded by other tones within a period of several hundred milliseconds.

Another approach to analyzing the neural encoding of stimulus sequences has been the presentation of complex stimuli, mostly animal vocalizations (Glass and Wollberg 1983; Steinschneider et al. 1994; Wang et al. 1995; for review see Newman 1988). These studies utilized voice onset time continua, time-reversed vocalizations, and time-compressed or -expanded calls. Results indicate that the responses of cortical neurons depend on temporal features of communication sounds, such as the voice onset time and the temporal separation and temporal order of individual parts of calls.

These two approaches have provided only limited systematic insight into the capability of cortical cells to code timevarying sounds. Presentation of spectrally uniform stimulus sequences, such as clicks or amplitude-modulated tones, uncovers how cortical cells respond to sequences of the same sound. These results, however, do not allow prediction of how cells respond to more complex sounds, either sequences of pure tones of different frequency and intensity or natural sounds like vocalizations. Analysis of the responses of cortical neurons with the use of animal vocalizations, on the other hand, has severe limitations in uncovering how neurons respond to vocalizations different from those tested or other time-varying sounds, because it is not clear what spectral and temporal features determine the neural sensitivity.

In an attempt to arrive at a more general understanding of how cortical neurons encode auditory sequences, in the present study we employed a forward masking paradigm. We used a pair of two pure tones that were varied in frequency, intensity, and temporal separation. In our experiments the changes in neural excitability induced by the first "masker" stimulus were analyzed with a constant "probe" stimulus, presented at various temporal intervals after the masker onset.

After completion of our experiments a study was published that also investigated nonsimultaneous two-tone interactions with the use of a similar stimulus paradigm (Calford and Semple 1995). In that study, the authors concentrated on the explanation of potential origins of cortical forward masking, e.g., the relation to sideband inhibition and to nonmonotonic rate-level functions. In contrast, the focus of the present study was on the time course of two-tone interactions. The dependence of the duration of two-tone interactions on the frequency and intensity was quantitatively assessed. To attempt a description of the spectrotemporal response properties of auditory neurons, characteristics of two-tone interactions were then related to various "static" receptive field properties of the neurons, including characteristic frequency $(\mathrm{CF})$, threshold, shape of rate-intensity functions, bandwidth, inhibitory subregions of the receptive field, and latent period of neural response. Recordings were performed both from single units and neural clusters, allowing an estimate of how characteristics of two-tone interactions are locally distributed. The present article describes the spectrotemporal characteristics of stimulus interactions for re- sponse inhibition and latency. The characteristics of forward facilitation will be described in a forthcoming article. Parts of the results have been published previously (Brosch and Schreiner 1994).

\section{METHODS}

\section{Surgery}

A total of 12 experiments was conducted on adult cats. The methods for surgical preparation, recording, and stimulation techniques were similar to those described previously (Schreiner and Mendelson 1990), and were conducted according to the rules for animal experimentation of the American Society for Neuroscience. In brief, cats were initially anesthetized with a mixture of ketamine $\mathrm{HCl}(10 \mathrm{mg} / \mathrm{kg})$ and acepromazine $(0.28 \mathrm{mg} / \mathrm{kg})$ given intramuscularly. An intravenous infusion line was placed and, for the following surgery, the cat received $\sim 30 \mathrm{mg} / \mathrm{kg}$ Nembutal (titrated to effect). Body temperature was measured with a rectal probe and maintained at $\sim 37.5^{\circ} \mathrm{C}$ by means of a heating pad. The trachea was incised in the intercartilaginous area and a tracheotomy tube was inserted. Thereafter the cat was placed in a head holder. A craniotomy was performed over the estimated location of the primary auditory cortex (AI) of the right hemisphere. The opening was further widened with rongeurs until the posterior ectosylvian sulcus was visible. The dura was removed and the cortical surface was protected with a film of silicone oil. For some of the electrophysiological recordings the oil was replaced with a thin layer of agarose to minimize brain pulsations. Anesthesia was maintained throughout the experiment by a continuous infusion of pentobarbital sodium $\left(1-2 \mathrm{mg} \cdot \mathrm{kg}^{-1} \cdot \mathrm{h}^{-1}\right)$ in lactated Ringer solution (1-2 $\left.\mathrm{ml} \cdot \mathrm{kg}^{-1} \cdot \mathrm{h}^{-1}\right)$. In two experiments we replaced the pentobarbital with Ketamine $\left(1-2 \mathrm{mg} \cdot \mathrm{kg}^{-1} \cdot \mathrm{h}^{-1}\right)$ and Diazepam $(1-2$ $\left.\mathrm{mg} \cdot \mathrm{kg}^{-1} \cdot \mathrm{h}^{-1}\right)$ after completion of the surgery. The infusion rate was adjusted according to several criteria that included electrocardiogram, body temperature, and the status of periodically checked reflexes. The cat received an injection of dexamethasone $(0.14 \mathrm{mg} /$ $\mathrm{kg} \mathrm{sc})$ to prevent brain edema, together with atropine $(1 \mathrm{mg} / \mathrm{kg})$ to reduce salivation.

\section{Neural recording}

One or two closely spaced tungsten microelectrodes (1-2 M $\Omega$ at $1 \mathrm{kHz}$ ) were advanced with a hydraulic microdrive (Kopf) to a depth 700-1,200 $\mu \mathrm{m}$ below the cortical surface at different locations of AI. The signals of the electrodes were band-pass filtered $(1-3 \mathrm{kHz})$ and amplified. Action potentials of single neurons or a small group of neurons were separated with triggering devices (Bak Dis-1 and Alpha-Omega Engineering, MSD). In a few occasions in which single- and multiunit activity were simultaneously recorded through the same electrode, the trigger level for the cluster was set such that the cluster recording did not include the action potentials of the single unit. Spike events were sampled with a resolution of $0.03 \mathrm{~ms}$ and stored in a computer (DEC 11/73 or IBM compatible). The recording window was in the range of 50 and $400 \mathrm{~ms}$ after each trial onset.

\section{Acoustic stimulation}

Experiments were performed in a double-walled sound-shielded chamber (IAC). Acoustic stimuli were generated by a microcomputer (TMS 32010 or TMS 320C30) at a sampling rate of 8 or 16 $\mu$ s with a dynamic range of $80 \mathrm{~dB}$. The signal was fed into an antialiasing filter $(55 \mathrm{kHz} ; 96 \mathrm{~dB} /$ octave $)$. Additional attenuation was provided by two passive attenuators (Hewlett-Packard or Tucker Davis, PA4). The attenuated signal was amplified and delivered via headphones (STAX 54) to both ears. Depending on 
the type of binaural interaction of the neuron under investigation, stimuli were presented to the contralateral ear, to the ipsilateral ear, or to both ears.

Excitatory receptive fields were assessed from the responses to 675 different bursts of pure tones. Stimuli had a duration of 30 or $50 \mathrm{~ms}$ when single tones were presented. The rise-fall time was 3 $\mathrm{ms}$. They were presented in a pseudorandom sequence every 380 $\mathrm{ms}$. For a few quickly adapting neurons the interstimulus interval was extended up to $500 \mathrm{~ms}$. Frequency of the tones was selected from 45 frequency values from a range of 3-5 octaves that was centered on the neuron's CF. Tone intensity covered a range of 70 $\mathrm{dB}$ with 15 intensity steps of $5 \mathrm{~dB}$. For the investigation of forward masking effects, a tone was selected from the receptive field that, if presented alone, evoked reliable neural responses. This tone, termed the probe tone, was usually at the $\mathrm{CF}$ and $10-20 \mathrm{~dB}$ above threshold.

Inhibitory sidebands of the receptive field could not be assessed from the presentation of single tones because neurons generally had low spontaneous activity. Therefore a simultaneous two-tone masking paradigm was employed. One of the two tone bursts was held constant while the other was changed for each presentation. The constant tone was at the neuron's CF and had an intensity that yielded a reliable response if presented alone (10-20 dB above threshold). The variable tone burst was varied over a frequency range of 3-5 octaves and over an intensity range of $70 \mathrm{~dB}$ in the same way when excitatory receptive fields were measured.

The time course of two-tone interactions was assessed by presenting a variable masker that was followed by a probe (Fig. 1). We parametrically varied the intensity and frequency of the masker and the temporal separation of the masker and probe. Similarly to the estimate of the excitatory receptive field, intensity of the masker varied in $5-\mathrm{dB}$ steps over a range of $70 \mathrm{~dB}$; frequencies covered a range of 3-5 octaves and 45 values were applied. Thus a total of 675 maskers at 45 frequencies at 15 intensities was presented. The temporal separation of the stimulus pair was measured with respect to the stimulus onsets and will be referred to as "stimulus onset asynchrony" (SOA) in the following. SOAs were in the range from 10 to $400 \mathrm{~ms}$. Because both the masker and the probe tone had a duration of $30 \mathrm{~ms}$ in the two-tone condition, the interval from masker offset to probe offset $(\Delta \mathrm{t})$ was always $30 \mathrm{~ms}$ shorter than SOA. Although the study focused on nonsimultaneous stimulus interactions, temporally partially overlapping stimuli were also tested in a few cases. The first SOA tested usually was 30 or 50 ms. Thereafter, other SOAs were presented in a random order. Depending on the duration of the two-tone interaction and on how long recordings from single neurons could be maintained, three to

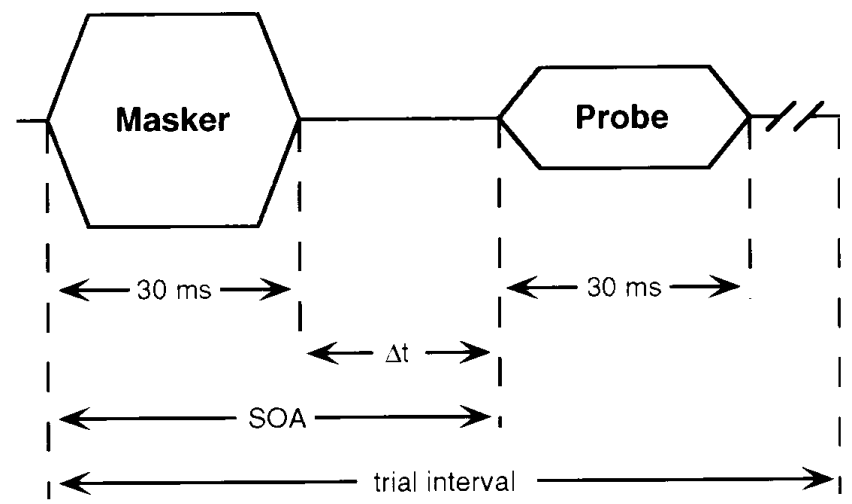

FIG. 1. Stimulus paradigm. Frequency and intensity of masker as well as stimulus onset asynchrony (SOA) of masker and probe were parametrically varied. Masker and probe duration as well as frequency and intensity of probe were not changed in most experiments. $\Delta \mathrm{t}$, interval from masker offset to probe offset. nine SOAs were tested per neuron or neural cluster. The effect of the masker on the excitability of the neuron was always tested with the same probe tone, which was usually a CF tone, 10-20 dB above its neural threshold. Between each stimulus pair there was a pause between 350 and $1,030 \mathrm{~ms}$. In a few cases, two-tone interactions were tested with longer masker durations, and with probes at a different intensity of frequency.

\section{Data analysis}

For each frequency-intensity combination of the variable tone, the number of neural discharges during the presentation of the first (and the 2nd) stimulus were plotted in two-dimensional boxes to obtain stimulus response areas (e.g., Fig. 2). If receptive fields were determined, the response during the presentation of the variable tone was analyzed. If the time course of two-tone interactions was determined, the response during the presentation of the probe was analyzed.

Figure 2 shows two examples of relatively sparse frequency response areas obtained with the two-tone paradigm. Figure $2 \mathrm{~A}$ displays the responses to various maskers (and thus provides another determination of the receptive field), and Fig. $2 B$ shows the responses to the following probe. Stimulus response areas, both for maskers and probes, often contained some noise, and receptive field borders were not sharply defined. The reason for this was because each stimulus was presented only once. We reduced the variability of stimulus response areas by the following procedure. For most points of the stimulus response areas, the neural response probability to tones that differed only slightly in frequency or intensity was almost the same. Therefore the responses to similar stimuli could be taken to estimate the average response strength to a particular region in the stimulus space. In practice, stimulus response areas were low-pass filtered: responses to tones of the same intensity but differing by one frequency value were weighted by 0.22 , and those differing by two frequency values by 0.11 . Responses to tones of the same frequency but $5 \mathrm{~dB}$ louder or softer were weighted by 0.17 . The response of the actual point was added to this weighted measure from the surround, and the resulting number was then divided by 2 to give an average spike count. Tests showed that shapes and sizes of filtered receptive fields and masking tuning curves (MTCs) were similar for a wide range of different weighting factors. Depending on the variability of the "raw" stimulus response areas, individual stimulus response areas were filtered up to three times.

In the two-tone paradigm, commonly many probe responses were reduced after the presentation of a certain set of maskers. We use the term "inhibition" in the following only to simplify the presentation and discussion of the results. It is clear, however, that the reduction of probe response magnitude can also be generated by other neural and nonneural mechanisms different from inhibitory postsynaptic potentials. The limits of the stimulus range that induced forward inhibition will be referred to as MTC. MTCs were defined by comparing each location of the stimulus response area with a reference value. As reference we took the average response strength that was evoked when only the probe was presented. This value could be estimated from each stimulus response area as those probe responses that followed the softest maskers. For these 135 stimuli ( 45 frequencies at the lowest 3 intensities), the mean number of spikes per probe and its SD were computed. Subsequently, points of stimulus response areas were defined as being suppressed by the masker where the number of spikes was below the mean value minus half the SD (shaded area in Fig. $2 C$ ). This criterion was taken because it revealed MTCs similar to the those determined by visual inspection of the stimulus response areas.

Excitatory receptive fields were further characterized by the following parameters. At $\mathrm{CF}$, rate-intensity functions were extracted. To increase the estimate of responses strength, functions were com- 

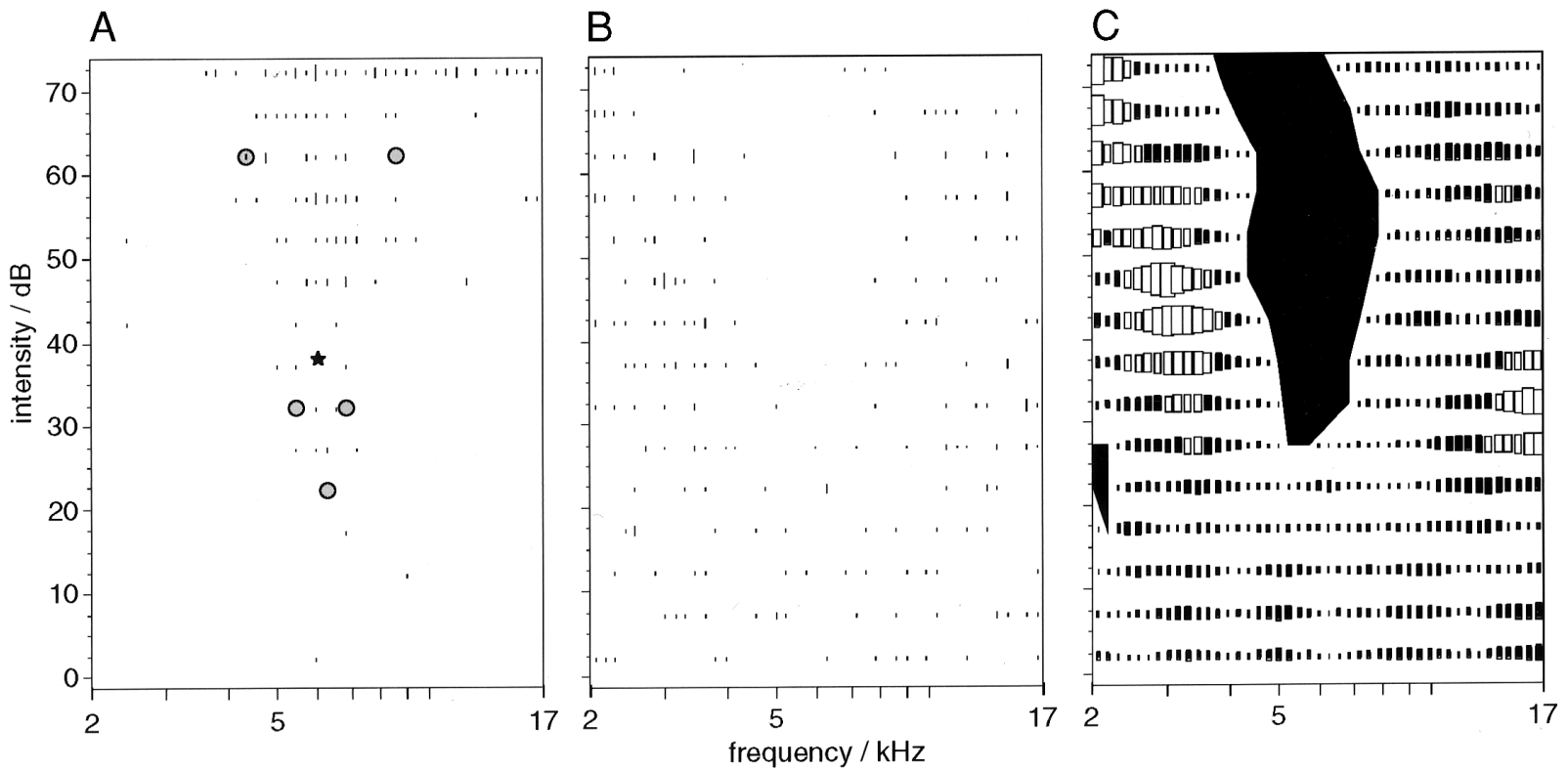

FIG. 2. Method used to assess the extent of masking tuning curves (MTCs). A: number of action potentials of a single cortical unit evoked by different frequency-intensity values of the masker. Six hundred seventy-five different maskers were presented, encompassing 45 frequency values at 15 intensities. The length of the shortest bar is equivalent to 1 action potential. Parameters to describe the extent of the receptive field were minimum threshold, characteristic frequency $(\mathrm{CF})$, and the lower and upper frequency borders 10 and $40 \mathrm{~dB}$ above the neuron's threshold $(O)$. Star: frequency and intensity of the probe that was used in $B$. $B$ : responses of a constant probe of $6 \mathrm{kHz}$ and $38 \mathrm{~dB}$ ( $\operatorname{star}$ in $A$ ). The probe was always preceded by a masker of variable frequency and intensity and the probe responses are plotted at the frequency-intensity location of the masker. $C$ : low-pass-filtered version of $B$, with weighting factors as described in METHODS. The size of each rectangle indicates the response probe strength after the presentation of a particular masker, weighted by responses to similar stimuli. Open rectangles: probe responses stronger than 3 times the mean response of the bottom 3 rows. Shaded areas: probe responses that were below the mean response of the bottom 3 rows minus half its SD.

piled by averaging responses for a CF tone and the two frequencies closest to CF (Schreiner et al. 1992). From this function, the transition point was defined as the point in the rate-intensity function that marked the change from a fast-growing, low-intensity portion to a less fast-growing, saturating, or decreasing high-intensity portion (Schreiner and Mendelson 1990). Monotonicity was defined as the slope of the rate-intensity function above the turning point and was measured in spikes per decibel. The third parameter was the shortest latency of the responses. It was obtained by determining the minimum in the latency-intensity profile at $\mathrm{CF}$ for the first spike latency (Schreiner and Raggio 1996). This profile was estimated from three stimulus frequencies at or near $\mathrm{CF}$ to eliminate spurious short-latency spontaneous events. Parameters of excitatory receptive fields were estimated from 4-10 stimulus repetitions, because the analysis also included responses to the first tone of the two stimuli.

\section{RES ULTS}

The results of this study are presented in three parts. The first part illustrates with three examples how masker stimuli of different frequencies and intensities affect neural responsiveness following the presentation of the masker. Three types of influence of a masker on the neural excitability are described. The second part reports general properties of the influence of different maskers and relates the temporal characteristics of neurons to their static receptive field properties. The presentation is concluded by briefly demonstrating the effects of two-tone interactions with stimulus pairs of different duration.

\section{Examples of MTCs}

Figure 3 gives an example of the temporal course of twotone interactions in AI. Temporal interactions were tested with 675 different masker conditions and six different SOAs of a fixed probe signal. The first stimulus of each pair (masker) varied in frequency and intensity, whereas the second stimulus (probe) was a constant $880-\mathrm{Hz}$ tone burst at an intensity of $63 \mathrm{~dB}$ SPL. In this and the following figures, raw data are displayed without additional low-pass filtering. The first frame shows for each frequency-intensity combination of the masker the number of neural discharges registered during the presentation of the masker. The length of the bars indicates the number of action potentials. Thus this plot shows the extent of the excitatory regions of the receptive field of the neuron. The other frames display the responses to the second stimulus of each pair, which was always constant in frequency and intensity. The response strength of the fixed stimulus is plotted at the location of each masker condition in the frequency-intensity plane. The resulting patterns reflect the interaction of the masker stimulus with the response of the probe. They show that neural response to the second stimulus depended on the frequency and intensity of the first stimulus. When the probe was presented immediately after the cessation of the masker ( 2 nd frame), the neuron did not respond to the probe for a wide range of masker frequency-intensity combinations. Such inhibition of probe responses was mostly seen when the neuron had already responded to the masker itself, i.e., when the masker 


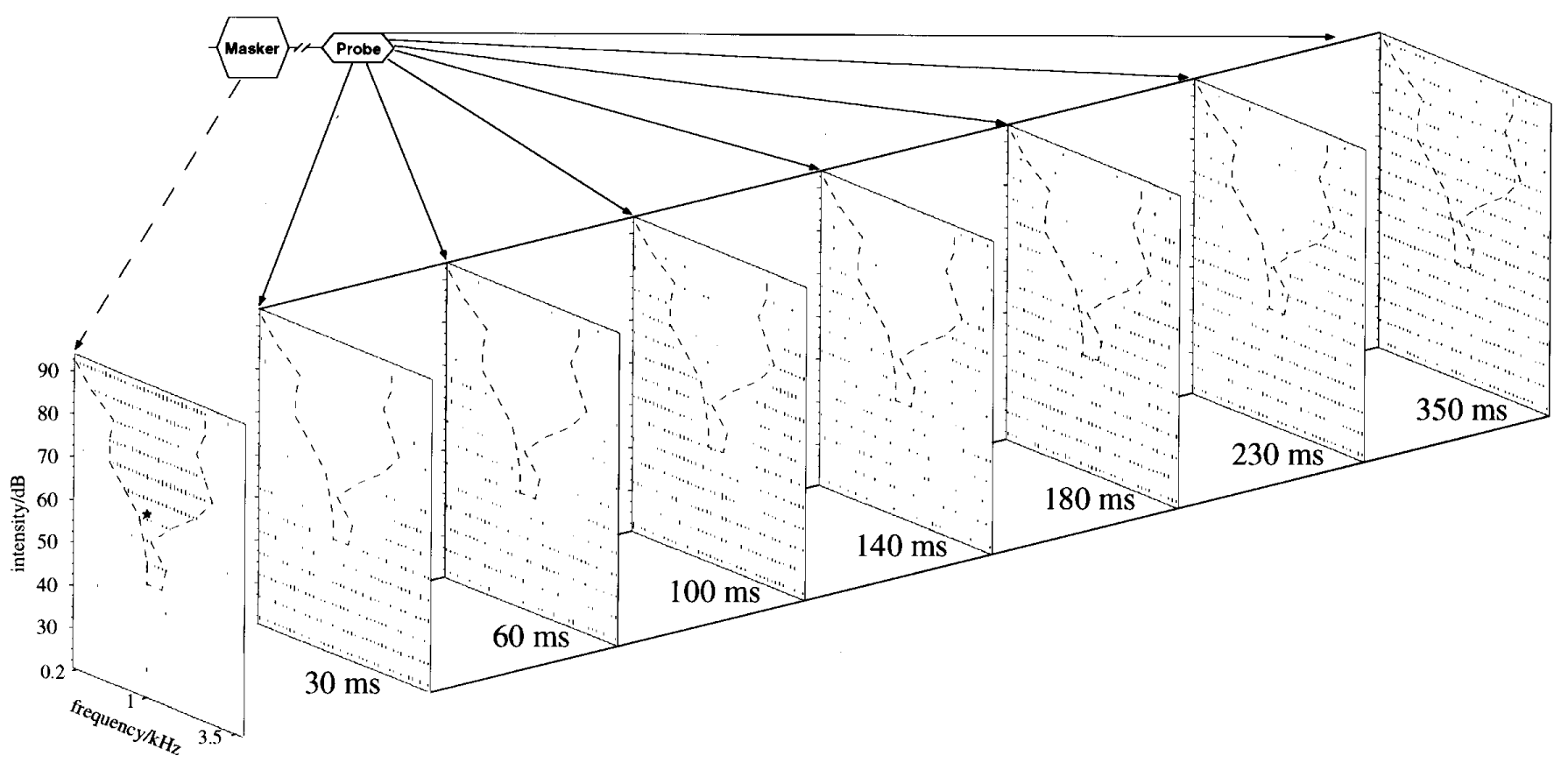

FIG. 3. Time course of 2-tone interactions for a broadly tuned neuron in the primary auditory cortex. First frame (free standing) of the pseudo-3-dimensional plot shows the neural responses during the presentation of the 1st tone (masker) of 675 different stimulus pairs. Dashed outline: receptive field border. Star: frequency and intensity of the 2nd tone of the pair (probe). The following, connected frames demonstrate the action of maskers of different frequency and intensity on the neural activity of the neuron. The effect of the masker was tested by registering the neural responses to a constant tone burst (probe). Six SOAs were presented (30-350 ms).

was within the excitatory receptive field. When the masker was well outside the receptive field of the neuron, probe responses were unaffected. Masker conditions near but outside the border of the excitatory receptive field also often inhibited the probe stimulus. This forward inhibition of neural activity lasted for up to several hundred milliseconds. The following frames demonstrate that the range of inhibiting maskers became gradually smaller as the temporal separation of masker and probe onsets was extended. Recovery from forward inhibition of the CF probe tone is first accomplished for maskers at the low- and high-frequency borders of the receptive field and for lowintensity maskers. Forward inhibition lasted the longest for masker frequencies at the center of the excitatory receptive field, i.e., around the neuron's CF.

A similar picture of action of maskers on the neural excitability was observed in other neurons. Figure 4 gives an example of a more narrowly tuned neuron with a nonmonotonic rate-level function, i.e., the neuron did not respond to loud tones. At the shortest SOA tested $(31 \mathrm{~ms})$, the neuron was inhibited from a wide range of maskers. The neuron not only failed to respond to the probe when it had already responded to the preceding masker, but also did not respond to probes when the masker was well beyond the borders of the excitatory receptive field. This indicates that forward inhibition can be generated by synaptic actions from other neurons and is not solely due to neural fatigue. As with the previously described neuron, the range of inhibiting masker conditions became gradually narrower when the temporal separation between masker and probe onsets was increased. Likewise, inhibition was induced the longest from masker frequencies around the neuron's CF. Complete recovery from forward inhibition was observed when the masker/ probe separation exceeded an SOA of $111 \mathrm{~ms}$. This neuron exhibited, in contrast to the first neuron, also another type of nonsimultaneous two-tone interaction. Inspection of the frames with an SOA of 51 and $91 \mathrm{~ms}$ reveals that the neuron responded with more action potentials to the probe when it was preceded by maskers of intermediate intensity and frequencies outside the receptive field. This forward facilitation was observed approximately in half of the neurons. Details will be reported in a forthcoming article.

Both inhibitory and facilitatory nonsimultaneous two-tone interactions could also be observed in multiunit recordings. Figure 5 shows an example of forward inhibition for a multiunit cluster. In general, characteristics of forward inhibition for multiunit clusters were similar to those seen for single neurons. MTCs for multiunit cluster, however, were mostly not as well defined as for single cells. The transition from stimuli that affected probe responses to stimuli where no effect on the neural activity could be assessed was more gradual and not as sharply defined as seen for single-unit responses. Thus the determination of MTCs for clusters was more sensitive to the criterion used for the definition of forward inhibition. The similarity of forward inhibition seen in single- and multiunit recordings indicates that adjacent neurons in the auditory cortex have several characteristics of forward inhibition in common. In the following paragraph we compare the spectrotemporal characteristics of forward inhibition of single units and multiunit clusters in more detail.

Maskers affected not only the magnitude but also the timing of probe responses, here assessed as onset latency. Figure 6 gives an example of this third type of action of maskers 


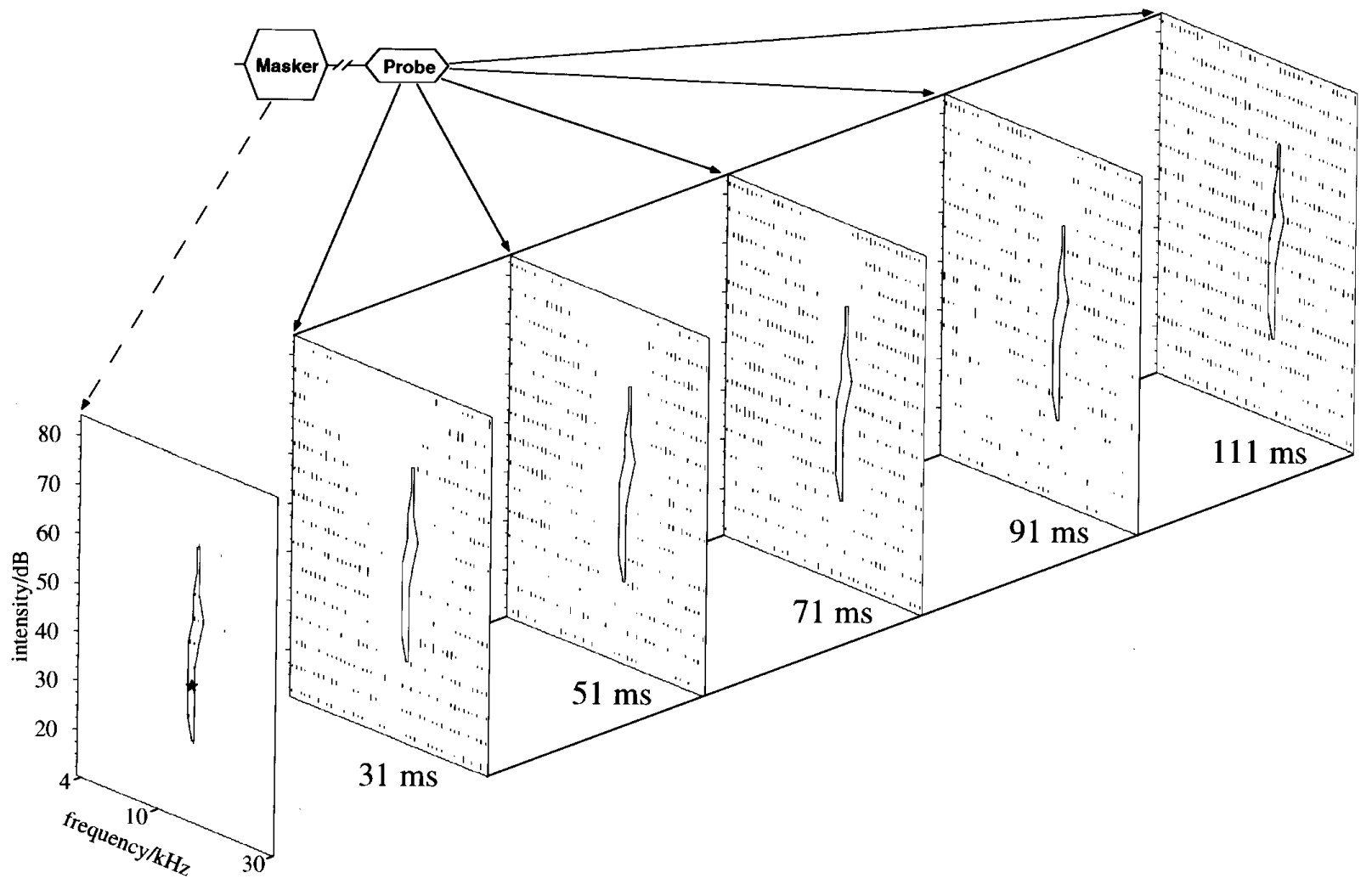

FIG. 4. Time course of 2-tone interactions for a narrowly tuned single neuron. Same conventions as in Fig. 3.

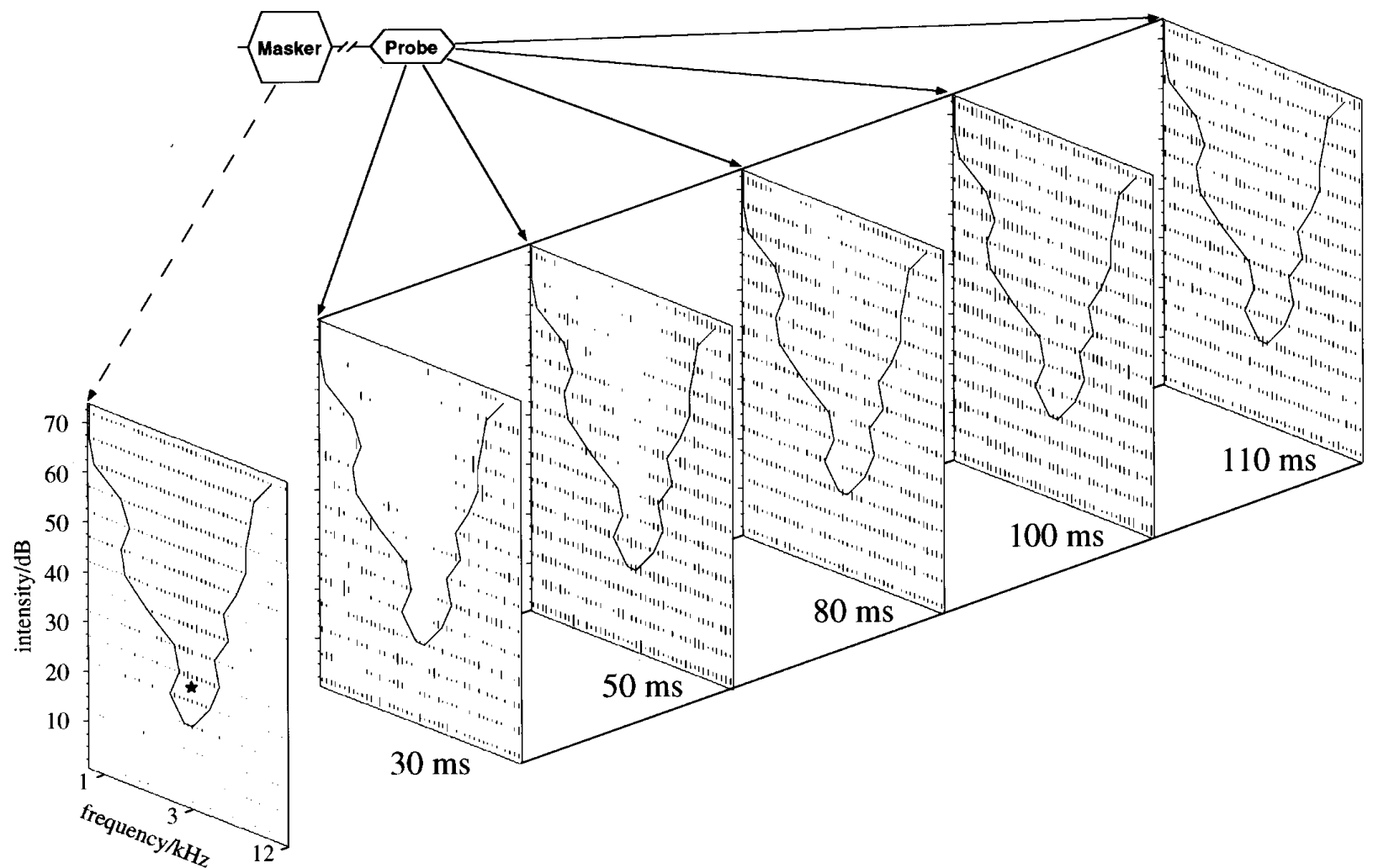

FIG. 5. Time course of 2-tone interactions for a multiunit cluster. Same conventions as in Fig. 3. 
A

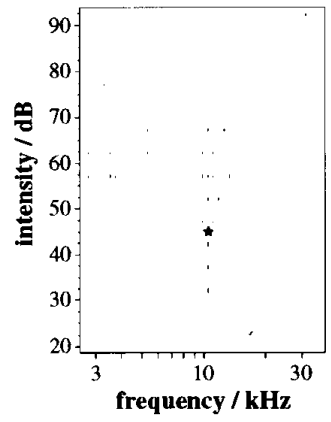

B

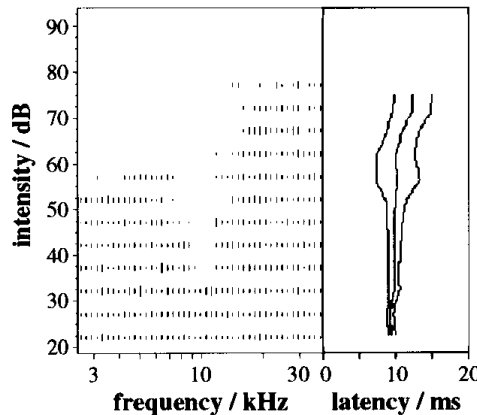

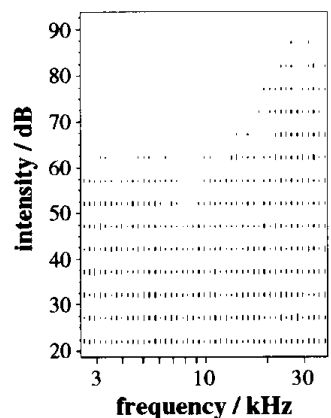

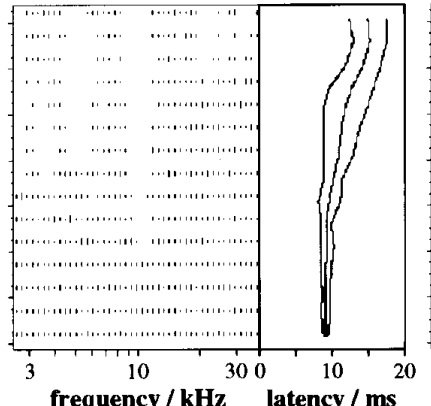

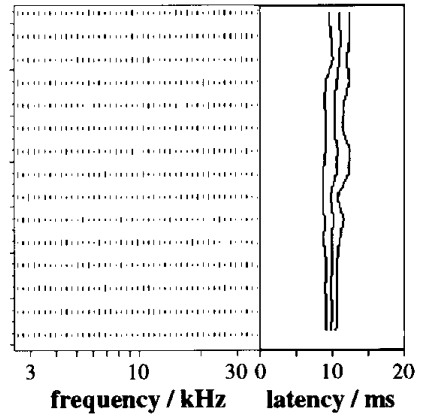

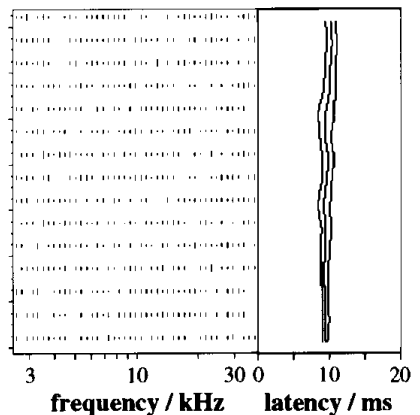

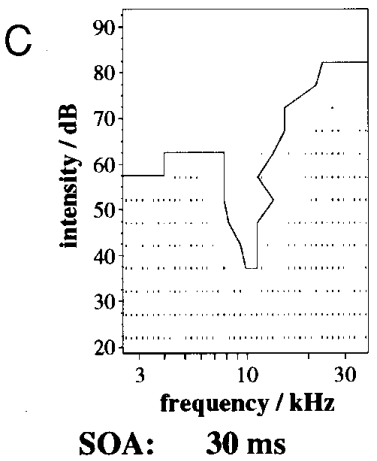
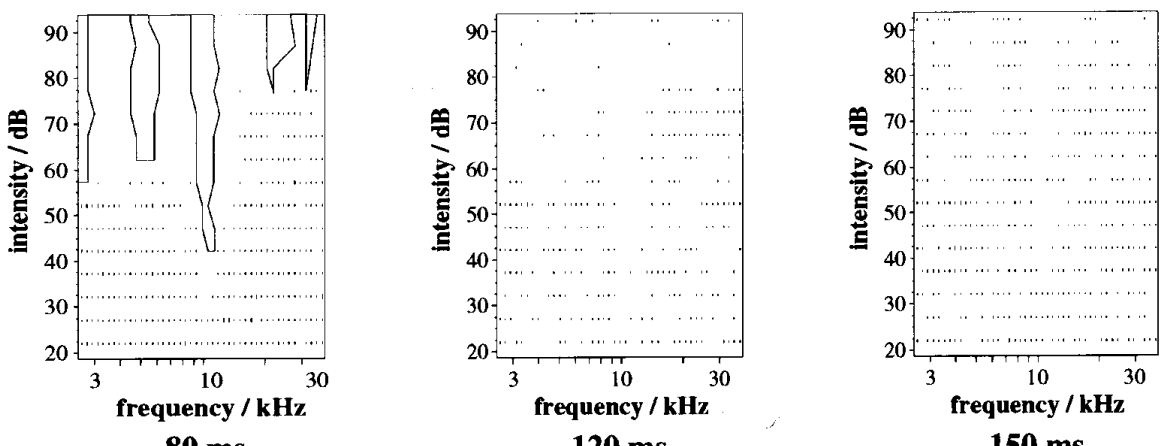

$80 \mathrm{~ms}$

$120 \mathrm{~ms}$

FIG. 6. Action of maskers on the latency of probe responses for single neurons. A: excitatory subfields of the receptive field (left) were obtained with individual tone bursts; inhibitory subfields ( $r i g h t$ ) were obtained with simultaneous presentation of a constant probe $(\star)$ and variable masker tone bursts. $B$ : time course of forward inhibition as reflected in the number of evoked action potentials throughout the course of the probe signal. The evoked action potentials were integrated over a range of 10-30 ms after the probe onset. Boxes to the right of each display: latency (mean $\pm \mathrm{SD}$ ) of the 1st action potential of the probe responses (averaged across different frequencies) against the intensity of the preceding masker. $C$ : time course of forward inhibition of probe responses measured over a short latency range (10-20 ms after probe onset). With long-latency responses omitted, the extent of the MTC increases. Shaded lines: MTCs for the long latency range $(B)$ for comparison.

on the neural activity. Here, the spectrotemporal course of forward inhibition is shown for two latency ranges of probe responses. Figure $6 B$ displays probe responses that occurred during the presentation of the probe (latency range 10-30 $\mathrm{ms}$ after the probe onset), whereas Fig. $6 \mathrm{C}$ shows the corresponding short-latency probe responses $(10-20 \mathrm{~ms})$. Comparison of stimulus responses areas for short- and long-latency ranges of the same SOA reveals two differences. Shortlatency probe responses were influenced from a broader frequency and intensity range of maskers than long-latency responses and, in addition, the influence of maskers lasted longer for short-latency responses.

Thus stimulus responses areas as shown in Fig. $6 B$ as well as those shown in Figs. 3-5 do not represent the entire range of masker conditions that affected the neural activity of the probe. They only indicate response magnitude reduction of the probe. Maskers lying outside the MTC could still have an influence on the timing of neural activity by prolonging the latent period of the probe responses. The increase in latency could even occur without any changes of the response strength to the probe. For most analyses of this study, MTCs were usually assessed by counting the total number of action potentials that occurred during the entire presentation of the probe, thereby ignoring the latency effects.

Latency shifts of probe responses could be demonstrated more quantitatively by generating plots of the latency of the first action potential of probe responses against the masker intensity. Examples are included in Fig. 6. The latency for a given intensity was calculated by averaging the latencies of probe responses that were registered after the presentation of up to 45 different masker frequencies of the same intensity. In our example, it was found that the latency of probe responses increased with the intensity of the preceding 
masker. The effect was very marked at an SOA of $80 \mathrm{~ms}$. The neuron, on average, responded $6.8 \mathrm{~ms}$ later after the presentation of maskers at $72 \mathrm{~dB}$ than after the presentation of the softest maskers ( $2 \mathrm{~dB})$. Latency differences were smaller for longer SOAs but, for this neuron, were still present at an SOA of $150 \mathrm{~ms}$.

An influence on the latency of probe responses was observed in most of the neurons of our study although effects were usually not as pronounced as in the example. We assessed latency shifts of probe responses by fitting linear regression lines to the intensity-latency functions for each SOA. Slopes were distributed in the range of a $-0.13-$ to 0.168 -ms increase in latency per decibel increase in masker intensity. Slope values were significantly higher at a SOA of $30 \mathrm{~ms}$ than at SOAs where no magnitude reductions of probe responses were evident. For SOAs $>30 \mathrm{~ms}$, no clear dependence of the latency on the masker intensity was seen for most of the neurons. At these time intervals the average latency varied, with a few exceptions, only weakly with the masker intensity.

We also explored whether the latency of probe response depended on frequency of the preceding masker. However, no systematic relation between both parameters was found.

\section{Quantitative description of the time course of forward inhibition}

For a quantitative description of the spectrotemporal dependence of forward inhibition, several parameters of MTCs were obtained for each SOA (Fig. 2). The main parameters were the lowest and highest inhibitory masker frequencies 10 and $40 \mathrm{~dB}$ (in a few cases $35 \mathrm{~dB}$ ) above minimum excitatory threshold, and the minimal and maximal intensities of the MTC that induced probe magnitude effects. Frequency values were expressed in octaves from the $\mathrm{CF}$ of the neuron. These values, together with the corresponding values of the excitatory receptive field, were plotted against the logarithm of SOA (Figs. 7 and 8).

Figure 7 shows the time course of the inhibitory frequency range for a sharply tuned neuron (Fig. 7, $A$ and $D$ ) and two more broadly tuned neurons at two masker intensities (10 and $40 \mathrm{~dB}$ above threshold, respectively). The time dependence of the frequency boundaries of MTCs could in most cases be well fitted with a linear regression line. This was possible for all three neurons at $40 \mathrm{~dB}$ above threshold (Fig. 7, $D-F$ ) but only for one of the neurons at $10 \mathrm{~dB}$ above threshold (Fig. 7B). Regression coefficients of all successful fits were in range of 0.03-0.99, with values measured at masker intensities $40 \mathrm{~dB}$ above threshold being higher than the corresponding $10-\mathrm{dB}$ values. The mean coefficients for lower and upper MTC boundaries were $0.66 \pm 0.28(\mathrm{SD})$ and $0.62 \pm 0.32$ at $10 \mathrm{~dB}$ above threshold, and $0.77 \pm 0.24$ and $0.74 \pm 0.25$ at $40 \mathrm{~dB}$ above threshold. Thus MTC size decreased exponentially with increasing interval between masker and probe onsets. For further characterizations of forward inhibition, two more parameters were measured from the time courses of MTCs: the lowest and highest inhibitory masker frequency at an SOA of $30 \mathrm{~ms}$ and at an SOA of three-fourths of the maximal duration of forward inhibition.

The intersection of the upper and lower regression lines revealed the maximal duration of forward inhibition 10 and
$40 \mathrm{~dB}$ above the neuron's threshold. The maximum of both values was taken as an estimate of the maximal duration of forward inhibition for a neuron. The intersection of the regression lines always occurred within the boundaries of the excitatory receptive field and was usually very close to the $\mathrm{CF}$ of the neuron. In other words, the longest inhibitory effects on a CF probe tone were caused by CF maskers. Only in a few cases was it necessary to assign a higher value for the maximal duration of forward inhibition than seen at 10 or $40 \mathrm{~dB}$ above threshold because of longer inhibitory periods for other masker intensities.

The data in the previous section indicate a strong dependency of the suppression-invoking frequency range on the time elapsed between masker and probe. A similar dependency can be seen for the effective range of masker intensities. The minimal and maximal masker intensity that induced forward inhibition were obtained at the $\mathrm{CF}$ of the neurons. Figure 8 shows examples of the time course of the intensity boundaries of MTCs at CF. For a short SOA, the minimal inhibitory masker intensity was mostly near or at the minimum excitatory threshold (Fig. 8, bottom gray lines). Although we tested maskers only over a limited intensity range of $70 \mathrm{~dB}$, it appeared that all $\mathrm{CF}$ tones more intense than the minimal inhibitory masker intensity inhibited probe responses. As the SOA was extended, different slope characteristics of the minimal inhibitory masker intensity were observed. Minimal inhibitory masker intensity could monotonically increase with SOA (Fig. 8A) or be constant over the entire intensity range for which inhibition of probe responses was observed (Fig. 8, $B$ and $C$ ). The temporal development of the maximal inhibitory masker intensity could not be determined as accurately because of the limited dynamic range of the maskers. With this limitation of the analysis in mind, neurons were found for which the loudest maskers inhibited probe responses over the entire duration for which forward inhibition was observed (Fig. 8, $A$ and $B$ ). However, there were also neurons for which a decrease of the maximal inhibitory masker intensity was clearly observed at longer SOA. Because of the different temporal profiles, the behavior of the minimal and maximal inhibitory masker intensity of MTCs was characterized for an SOA of $30 \mathrm{~ms}$ and for an SOA three-fourths of the maximal duration of forward inhibition ( see below).

\section{General properties of forward inhibition}

The properties of forward inhibition were obtained for a total of 53 single neurons and 58 multiunit clusters in AI. The CFs of the neurons were between 0.9 and $15.4 \mathrm{kHz}$ (Fig. 12C). The ranges of latencies, thresholds (Fig. 12A), bandwidths (Fig. 12D), and nonmonotonicities were similar to those found in previous reports of the spatial distributions of these parameters in cats (e.g., Schreiner and Mendelson 1990; Schreiner et al. 1992). This indicates that most portions of the dorsoventral extent of isofrequency domains were encountered in the present study. Forward inhibition of probe responses was found in 107 cases according to the definition given in METHODS. The criterion for forward inhibition failed for four neurons because neurons did not respond to the probe when it was preceded by maskers at low intensities. Responses of these neurons, however, were 

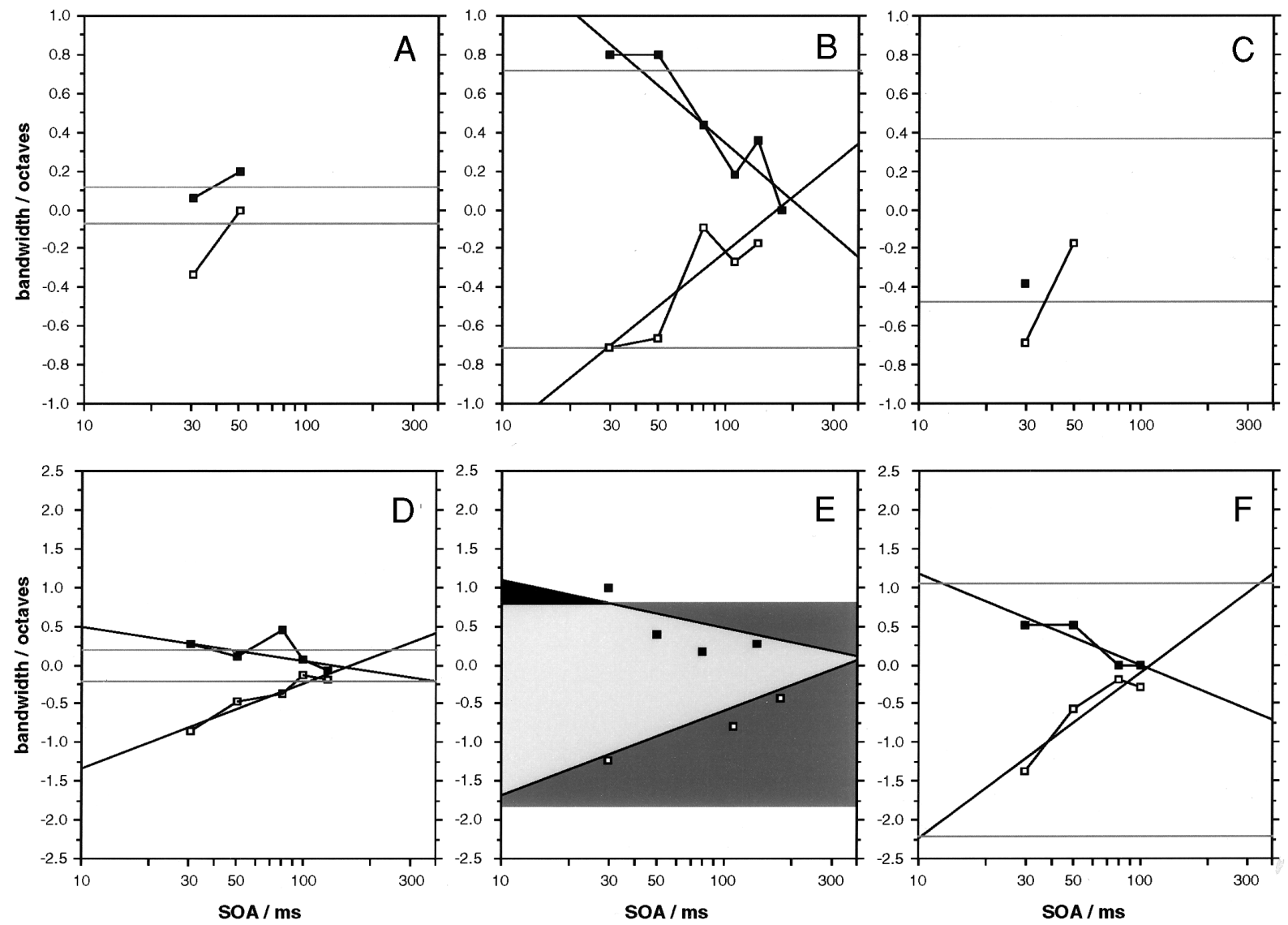

FIG. 7. Time courses of the frequency range of inhibitory maskers for 3 neurons. Abscissa: temporal interval between masker and probe onsets (SOA). Ordinate: distance of the lowest ( $\square$ ) and highest ( $\mathbf{m})$ inhibitory masker frequency from the neuron's $\mathrm{CF}, 10 \mathrm{~dB}(A-C)$ and $40 \mathrm{~dB}(D-F)$ above the excitatory threshold. Values were fitted with a linear regression analysis. Regression coefficients for the high- and low-frequency slope were 0.81 and $0.63(B), 0.45$ and $0.91(D), 0.10$ and $0.74(E)$, and 0.80 and $0.87(F)$, respectively. Borders of the excitatory receptive field at the same intensities are indicated by the horizontal lines. Shadings in $E$ correspond to 4 different masker-probe interactions. Light gray: probe response is completely suppressed by preceding excitatory masker response. Black: probe is completely suppressed but not preceded by an excitatory masker response. Dark gray: probe magnitude is not suppressed by preceding excitatory response. White: probe is not suppressed and not preceded by excitatory masker response. Diagonal lines: linear regression of the upper and lower frequency boundaries of the inhibited area.
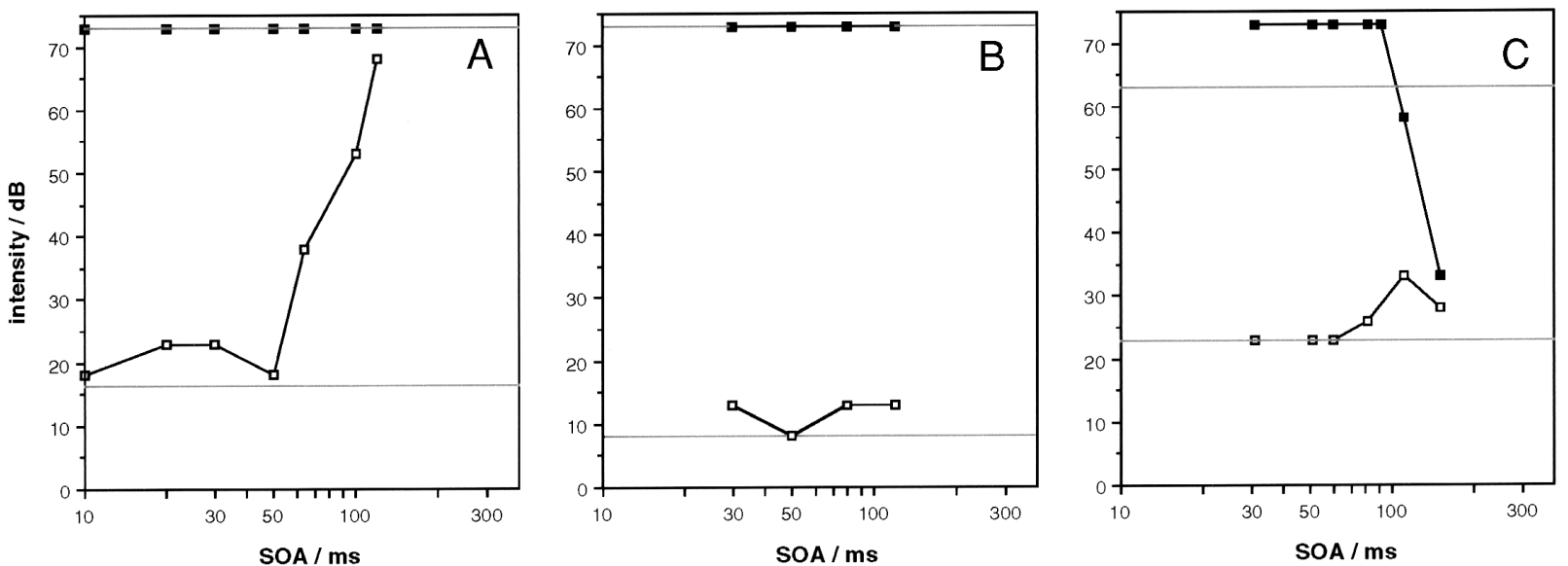

FIG. 8. Time courses of the intensity range of inhibitory maskers for 3 neurons. Ordinate: minimal inhibitory masker ( $\square$ ) and the maximal masker intensity $(\square)$ that inhibited probe responses within the dynamic range tested. Gray horizontal lines: intensity range over which the neuron responded with an excitatory response. In $A$ and $B$, the highest tested intensities resulted in a response. 
facilitated by particular maskers, and thus also exhibited nonsimultaneous two-tone interactions. Thus responsiveness of all neurons in AI depended on the temporal stimulus context.

Forward inhibition usually was induced from a single, continuous frequency-intensity range of maskers. Only in a few cases, several spatially separated inhibitory areas were found at individual SOAs (e.g., Fig. 6). Mostly, MTCs were $\mathrm{V}$ shaped, i.e., the frequency range of inhibiting maskers increased with the masker intensity. For an interval of 30 ms between the onsets of masker and probe, MTC bandwidths $40 \mathrm{~dB}$ above the neuron's threshold were, with one exception, larger than respective bandwidths $10 \mathrm{~dB}$ above threshold. For longer SOAs, occasionally diamond shaped or nonmonotonic MTCs were observed. Within the MTC, probe responses were totally abolished, whereas at the borders of the MTC, probe responses could be partially suppressed. A more precise description of this transition zone was, however, not possible because each stimulus pair was presented only once.

The widest range of maskers inducing forward inhibition was seen for the shortest delay between masker and probe onsets, i.e., when the probe was presented immediately after the cessation of the masker $(\mathrm{SOA}=30 \mathrm{~ms})$. MTCs became smaller when the temporal separation of masker and probe was increased. For seven of eight neurons tested at SOAs of 30 and $40 \mathrm{~ms}$, the bandwidth with the shorter SOA exceeded that for the longer SOA. Comparison of inhibitory areas at an SOA of $30 \mathrm{~ms}$ with an SOA of $50 \mathrm{~ms}$ revealed that 55 of 60 neurons had a greater MTC bandwidth at the shorter SOA. The intensity range of MTCs was, on average, constant for these SOAs. For SOAs $<30 \mathrm{~ms}$, i.e., with masker and probe partially temporally overlapping, stimulus response areas were more difficult to interpret. On the one hand, simultaneous interactions between masker and probe were superimposed on the nonsimultaneous stimulus interactions. Neural responses to the masker were, on the other hand, temporally overlapping with probe responses, which did not allow analysis of stimulus pairs separated by $<20$ ms. Overlap of neural responses no longer affected maskerprobe interactions for an SOA of $20 \mathrm{~ms}$. Compared with an SOA of $30 \mathrm{~ms}$, no overall difference of MTCs was found, either for the bandwidth $40 \mathrm{~dB}$ above the neuron's threshold or for the minimal inhibitory masker intensity $(n=19)$.

For SOAs $>30 \mathrm{~ms}$, forward inhibition was induced from a smaller frequency and intensity range of the masker. Figure 9 summarizes how various parameters of MTCs change from a 30-ms SOA to an SOA at three-fourths of the maximal duration of forward inhibition. The center frequency of the MTC, i.e., the masker frequency with the lowest intensity producing forward inhibition, matched very closely for the two considered SOAs (Fig. 9A). Only in 14 of 73 cases was the center frequency shifted with increase in SOA, and with the exception of 1 case, it shifted to a slightly lower frequency. The temporal development of intensity range of inhibitory maskers could be determined reliably only for the minimal inhibitory masker intensity, which almost always was found within the tested dynamic range. It usually was the lowest for a 30-ms SOA and remained constant for most neurons for SOAs up to a certain value beyond $30 \mathrm{~ms}$. Then, louder maskers were necessary to inhibit the neural activity.
At an SOA of three-fourths of the maximal duration of forward inhibition, minimal inhibitory masker intensity increased for 64 of 73 neurons compared with respective values at $30 \mathrm{~ms} \mathrm{SOA} \mathrm{(Fig.} \mathrm{9C).} \mathrm{At} \mathrm{short} \mathrm{SOAs,} \mathrm{forward} \mathrm{inhibi-}$ tion was induced from all maskers louder than the minimal inhibitory masker intensity. For longer SOAs, probe responses of several neurons were only inhibited from maskers up to a moderate intensity, whereas a very loud masker did not induce forward inhibition. At an SOA of three-fourths of the maximal duration of forward inhibition, 25 of 80 neurons were not inhibited from the most intense masker that was tested. The actual percentage of neurons showing nonmonotonic suppression-level functions may be underestimated because maskers were only presented over a limited dynamic range.

The frequency range of forward inhibition decreased as the interval between masker and probe onsets was increased in all but one case (Fig. 9B). The lowest inhibitory masker frequencies increased with SOA and the highest inhibitory masker frequencies decreased with SOA. As pointed out in the examples shown in Fig. 7, for many neurons the shift of the upper and lower frequency boundaries (measured in octaves) of the MTC was proportional to the logarithm of SOA. The lower-frequency boundary of the MTC increased significantly $(n=54, Z=-4.76, P<0.001)$ faster with SOA $(2.26 \pm 1.65$ octaves of masker frequency per decade of SOA) than the higher-frequency boundary of the MTC decreased with SOA $(-1.24 \pm 0.83$ octaves/decade $)$. As mentioned above, the frequency of the minimal inhibitory masker intensity did not change with SOA for the majority of neurons.

Maximal duration of forward inhibition lasted between 53 and $430 \mathrm{~ms}$, with an average of $143 \pm 71 \mathrm{~ms}$ (Fig. 10). There was no difference between the single-unit and multiunit samples $\left(\chi^{2}=0.016, P=0.90, n=94\right)$. The longest inhibition was induced, in most cases, from maskers around the neuron's CF. Maskers that induced the longest forward inhibition usually had a high intensity. However, for a number of cases maskers of an intermediate intensity were more effective, suggestive of nonmonotonic suppression-level functions. Forward inhibition was shorter lasting for softer maskers and maskers with frequencies different from $\mathrm{CF}$. The dependence of the frequency of the masker can be demonstrated with an example (Fig. $7 E$ ). If the masker had a frequency similar to the $\mathrm{CF}$ of the neuron and had an intensity $40 \mathrm{~dB}$ above neural threshold, the neuron was inhibited for a period of $430 \mathrm{~ms}$. For a different masker frequency just 1 octave below the $\mathrm{CF}$, forward inhibition only lasted for $44 \mathrm{~ms}$. That means that the neuron responded to each tone of a stimulus pair if the frequencies of the two tones were different, whereas it responded to the first tone only when the tones were the same. The average dependence of forward inhibition duration on the frequency difference of the masker and the probe is displayed in Fig. 11. It was calculated from the decrease of the frequency range of MTCs $40 \mathrm{~dB}$ above excitatory threshold and the maximal duration of forward inhibition.

\section{Comparison of MTC and receptive field for short SOAs}

This and the following section compare spectrotemporal properties of forward inhibition and static receptive field 

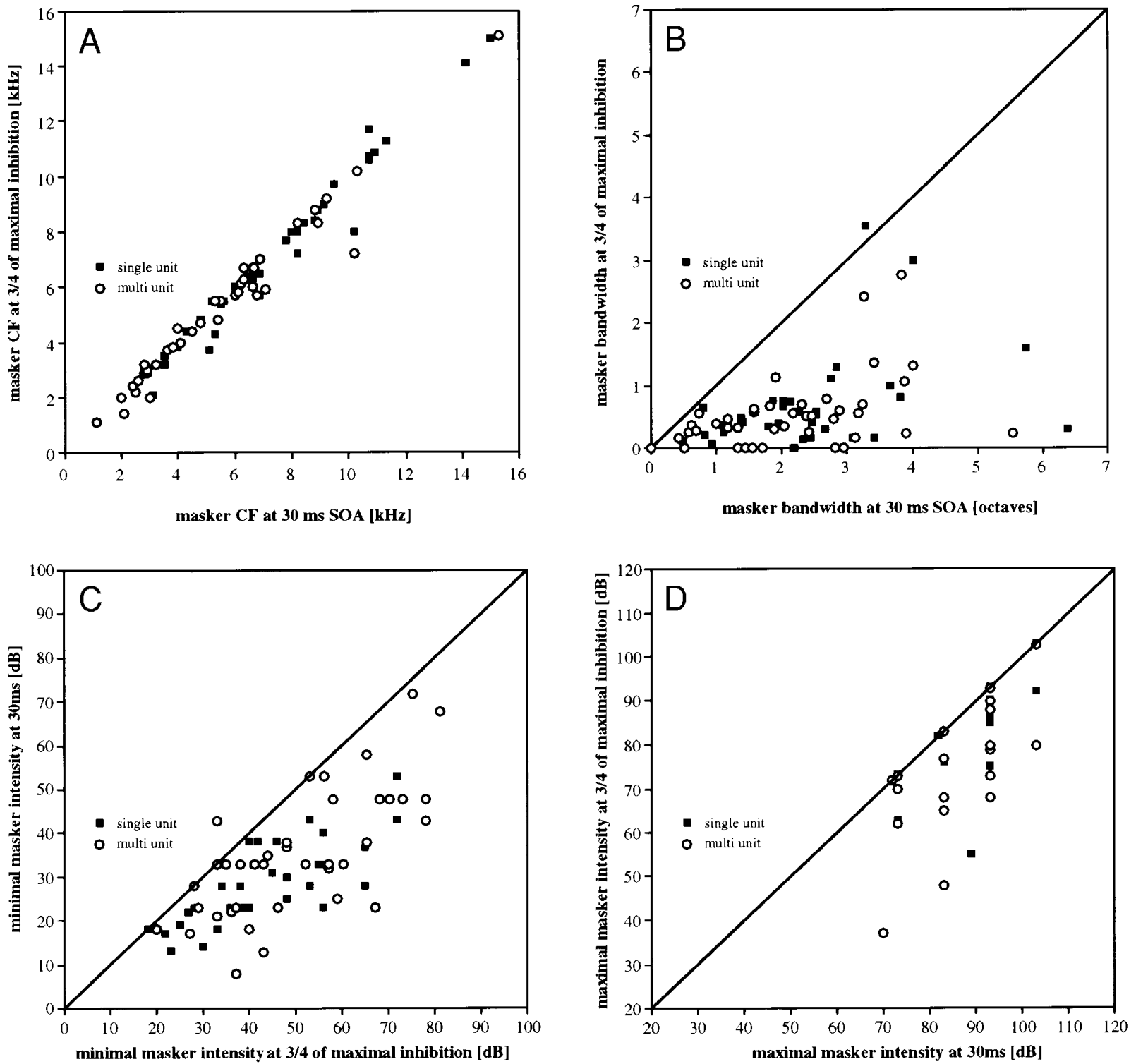

FIG. 9. Group results of recovery from forward inhibition. Each plot shows a parameter of the MTC at an SOA of 30 $\mathrm{ms}$ on the abscissa and the corresponding parameter at an SOA of $3 / 4$ of the maximal duration of forward inhibition on the ordinate. $A$ : frequency of the minimal inhibitory masker. $B$ : bandwidth of the inhibitory frequency range $40 \mathrm{~dB}$ above neural threshold. $C$ : minimal inhibitory masker intensity. Some dots indicate several data points.

properties. We first compared various receptive field parameters with corresponding parameters of MTCs at $30 \mathrm{~ms}$, at which the most widespread forward inhibition was induced (Fig. 12). In general, MTCs were centered on and scaled to the receptive field of a neuron. Neurons with large receptive fields had large MTCs and vice versa. Minimal inhibitory masker intensity was slightly $(6 \mathrm{~dB}$ for single units and $1 \mathrm{~dB}$ for multiunits) below the neural threshold. Differences were only significant for single units (Wilcoxon test, $n=43, Z=-3.91, P=0.0001)$. MTCs were, at this SOA, always $\mathrm{V}$ shaped, irrespective of the shape of the receptive field. The frequency of the minimal inhibitory masker intensity was similar to the $\mathrm{CF}$ of the neuron, both for single units $(n=43, Z=-1.222, P=0.222)$ and multiunits $(n=$ $51, Z=-0.769, P=0.442$ ). The bandwidth of MTCs 40 $\mathrm{dB}$ above neural threshold grew monotonously for louder maskers and was, on average, larger than corresponding values of the excitatory regions of the receptive field. The difference was highly significant for single-unit activity $(n=30$, $Z=-4.19, P<0.0001)$ and the same tendency was seen for multiunit activity $(n=30, Z=-1.74, P=0.08)$. In addition, a small asymmetry of MTCs was seen: MTCs extended 0.5 octaves further to the low-frequency site than the receptive field, whereas the difference was 0.3 octaves on the high-frequency site $(n=60, Z=-3.680, P=$ 0.0002 ). The width of MTCs at an SOA of $30 \mathrm{~ms}$ was also compared with the extent of the total receptive field $40 \mathrm{~dB}$ above neural threshold. Total receptive fields included both excitatory and inhibitory subfields. Inhibitory subfields were measured for 23 single units by simultaneously presenting a probe tone (see METHODS). Almost half of the neurons had MTCs that extended beyond the total receptive field. 


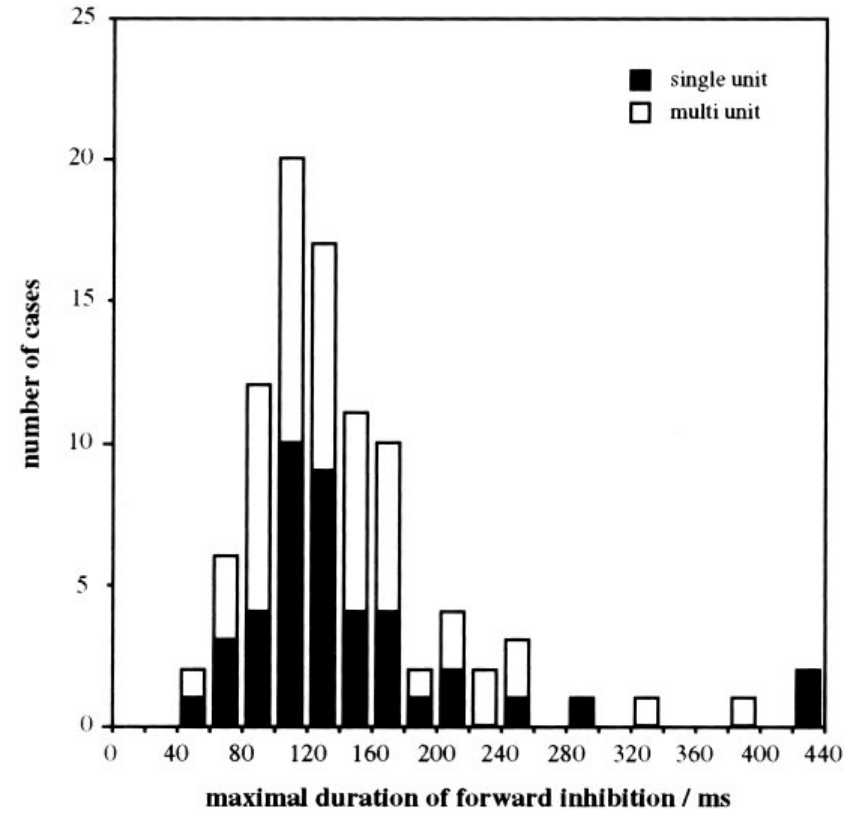

FIG. 10. Maximal duration of forward inhibition. For each single-unit and multiunit recording, the maximal duration of forward inhibition was obtained. Mode: 120 ms. Mean: $143 \pm 71$ (SD) ms.

This indicates that forward inhibition was not only induced from unequivocal excitatory and inhibitory regions of the receptive field, but, for the majority of neurons, even from stimuli outside the classical receptive field. However, on average there was no difference between both the lower $(Z=$ $0.00, P=1)$ and upper $(Z=-1.00, P=0.32)$ frequency limits of the MTC and the respective values of the total receptive field.

\section{Relation of temporal characteristics of forward inhibition to static receptive properties}

Several temporal characteristics of forward inhibition were compared with the static receptive field parameters by performing a correlation analysis (Table 1). Temporal characteristics included maximal duration of forward inhibition, decrease of frequency range of MTCs $40 \mathrm{~dB}$ above neural threshold, and change of the minimal and maximal inhibitory masker intensity between $30-\mathrm{ms}$ SOA and three-fourths of the maximal duration of forward inhibition. For the latter parameter two groups were compared. The first group consisted of neurons in which forward inhibition was not induced from loud maskers at three fourths of the maximal duration of forward inhibition. In the second group no change of the maximal inhibitory masker intensity was observed. Static receptive field parameters included $\mathrm{CF}$, bandwidth $40 \mathrm{~dB}$ above neural threshold (measured in octaves), neural threshold, turning point, nonmonotonicity of intensity-rate functions, and latency of first discharges.

Correlation coefficients were generally small, but in several instances statistically significant, indicating that spectral and temporal sensitivity of cortical neurons were to some degree linked. Along the tonotopic gradient of the auditory cortex, it was found that forward inhibition lasted longer for neurons with a low CF than for neurons with a high CF.
That means that forward inhibition lasted, on average, 72 ms longer for neurons with a $\mathrm{CF}$ of $1 \mathrm{kHz}$ than for neurons with a $\mathrm{CF}$ of $8 \mathrm{kHz}$. For neurons with low $\mathrm{CFs}$, the longest forward inhibition was frequently induced from maskers at intermediate intensities, whereas for neurons with high CFs, usually the longest forward inhibition was induced from more intense maskers. Characteristics of forward inhibition were related to other receptive field properties as well. Neurons with broad receptive fields usually were forward inhibited for the longest periods. By contrast, sharply tuned neurons showed shorter duration of inhibition. In addition, the decrease of widths of MTCs was more pronounced for broadly tuned neurons than for sharply tuned neurons. The minimal inhibitory masker intensity changed only a little as the interval between masker and probe onsets was increased.

The latency of responses to individual stimuli also was linked with several characteristics of forward inhibition. It was found that forward inhibition, on average, was shorter lasting for neurons with short latencies than for neurons with long latencies. Neurons that responded with long latencies at long SOA were no longer inhibited from maskers at high intensities.

\section{Forward inhibition is affected by stimulus duration}

The results presented so far were obtained with stimulus pairs of short duration $(30 \mathrm{~ms}$ each for masker and probe tone). However, the properties of forward inhibition could depend on other parameters of the two tones as well. Specifically, the duration of the masker could have a strong influence on forward inhibition. Figure 13 shows an example for two-tone interactions tested with

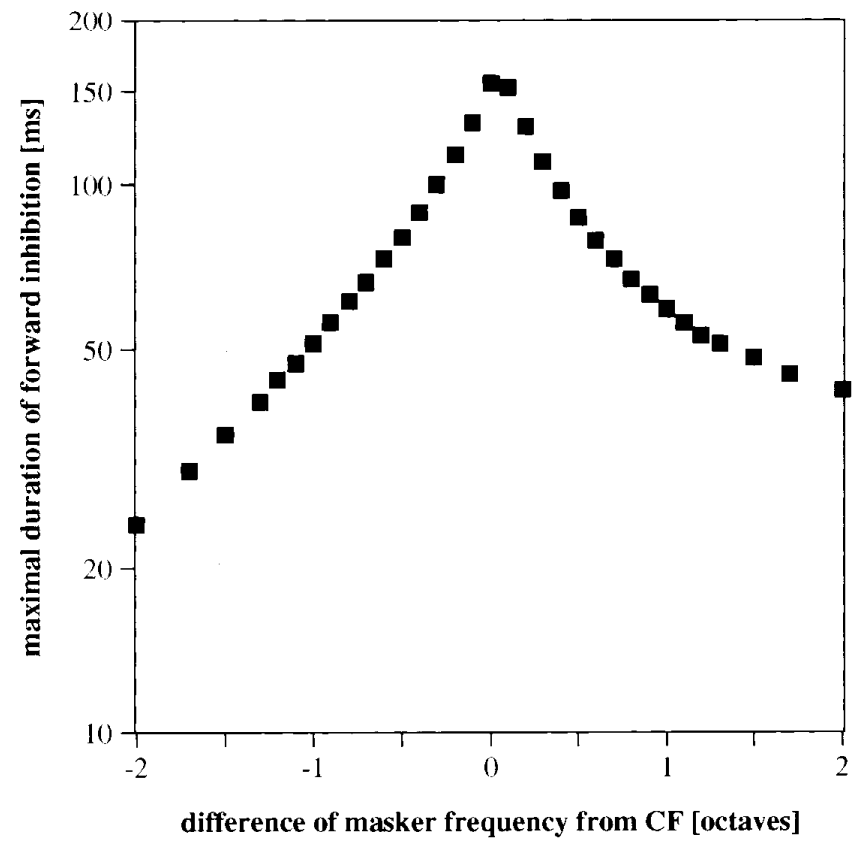

FIG. 11. Dependence of the duration of forward inhibition on the frequency separation of the masker and the CFs $40 \mathrm{~dB}$ above neural threshold. Positive and negative values: masker frequencies above and below $\mathrm{CF}$, respectively. Positive values were based on 61 cases and negative values on 58 cases. The maximal duration of forward inhibition for masker and probe of identical frequency was slightly higher than for the larger sample displayed in Fig. 11. 

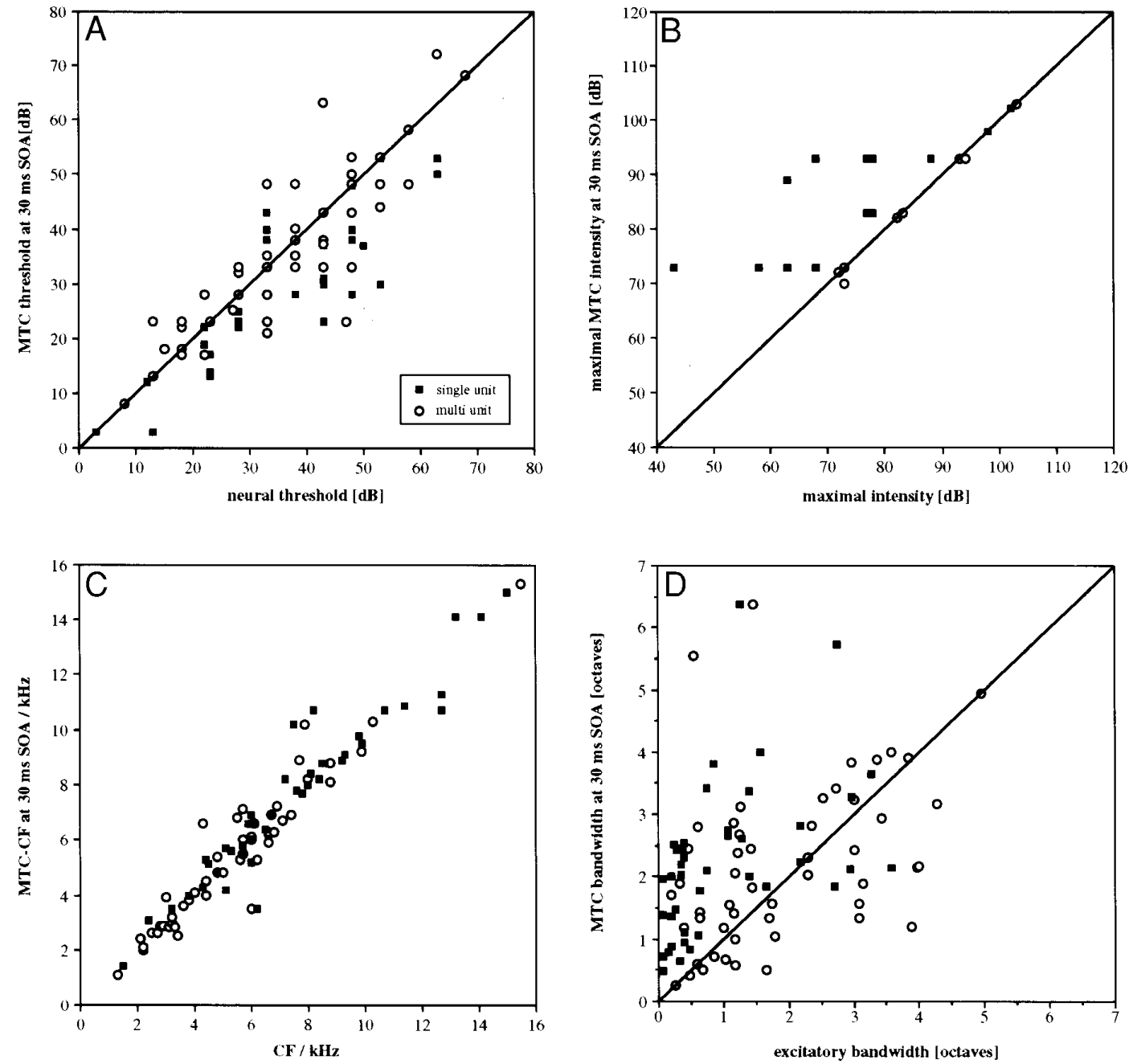

FIG. 12. Comparison of the extent of the receptive field of the neurons (abscissa) with the stimulus range of forward inhibition at an SOA of $30 \mathrm{~ms}$ (ordinate). A: minimal inhibitory masker intensity vs. neural threshold. $B$ : maximal inhibitory masker intensity vs. maximal intensity to which the neuron responded. $C$ : frequency of the minimal inhibitory masker vs. CF. D: bandwidth of MTCs $40 \mathrm{~dB}$ above neural threshold vs. excitatory bandwidth.

maskers of different duration. When the probe followed immediately after the cessation of a masker of short duration $(30 \mathrm{~ms})$, the neurons were inhibited from a broad range of maskers (Fig. 13B). The MTC was approximately of the size of the excitatory receptive field. For this cluster, forward inhibition lasted for $185 \mathrm{~ms}$ such that no effect of maskers on the neural activity was observed when probes were presented $190 \mathrm{~ms}$ after the onset of the masker (Fig. 13C). However, when the masker duration was prolonged to $190 \mathrm{~ms}$ and the effect on the neural activity was tested at the same SOA of $190 \mathrm{~ms}$, inhibition of several probe responses was observed (Fig.

TABLE 1. Relation of temporal charactersitics of forward inhibition and various receptive field properties

\begin{tabular}{|c|c|c|c|c|}
\hline $\mathrm{CF}$ & $-24 \mathrm{~ms} /$ octave* & & & $*$ \\
\hline Turning point & & & $-0.21 \mathrm{~dB} / \mathrm{dB} \dagger$ & \\
\hline Monotonicity & & & & \\
\hline Latency & $7 \mathrm{~ms} / \mathrm{ms} \dagger$ & $0.17 \mathrm{~ms} /($ octave $\times$ decade $) \dagger$ & & $\dagger$ \\
\hline
\end{tabular}

Parameters were subjected to pairwise regression analysis. Numbers: significant slopes. Significant correlation coefficients were in the range of 0.23 and 0.38 . CF, characteristic frequency. $* P<0.05 . \dagger P<0.01$. 

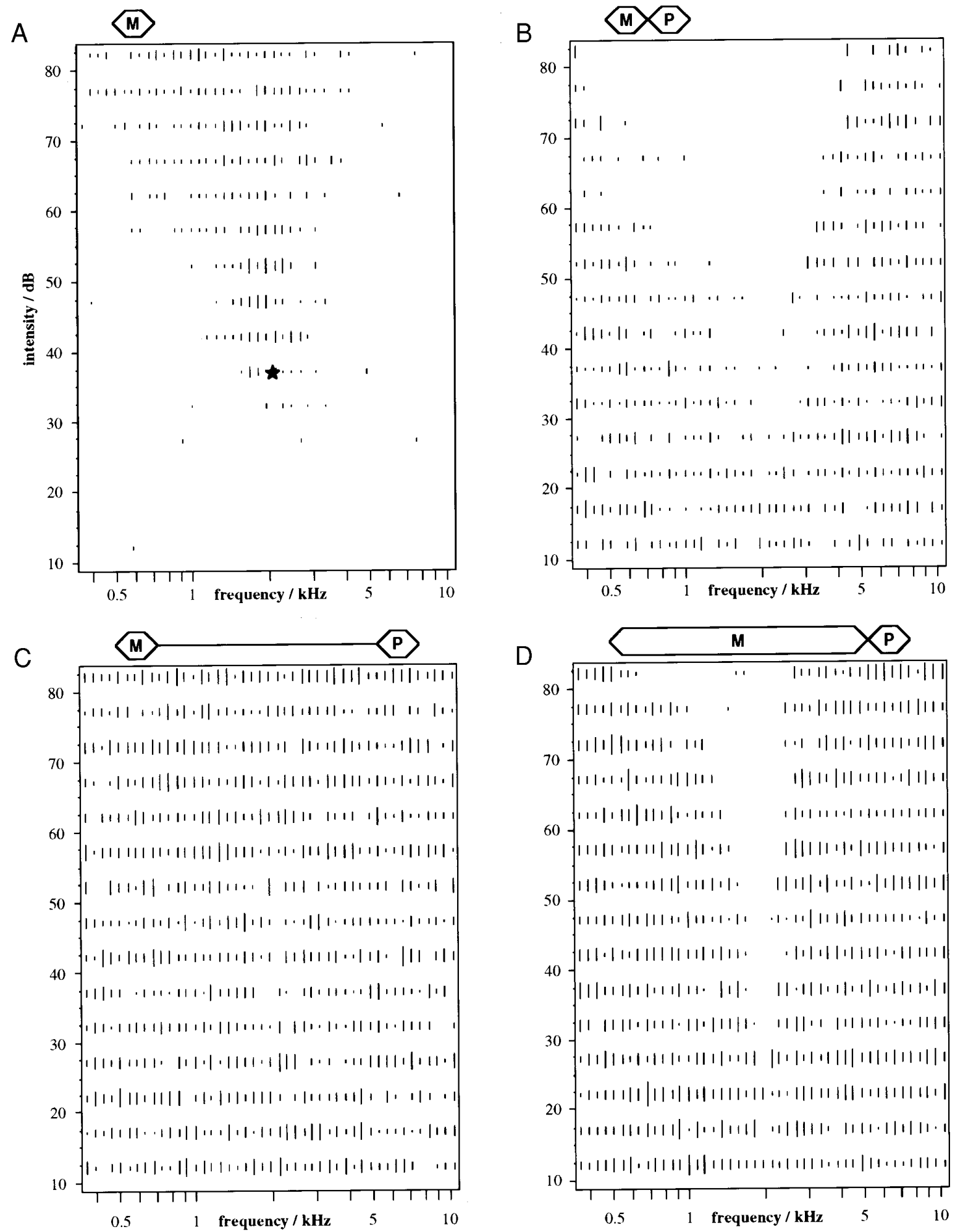

FIG. 13. Action of maskers of different duration on probe responses. A: neuron's receptive field and the location of the probe $(2 \mathrm{kHz}, 37.5 \mathrm{~dB} ; \star) . B-D$ : forward inhibition for different SOA and probe delays. $B$ and $C$ : probe responses after the presentation of short maskers (30 ms in duration) at 2 SOAs (30 and $190 \mathrm{~ms}$, respectively). $D$ : probe responses are displayed after the presentation of a long masker and at a long SOA (both $190 \mathrm{~ms}$ ).

$13 D$ ). Similar results were found in four other neurons ( 2 single units and 2 multiunits) where the masker duration was altered. This clearly demonstrates that forward inhibition reflects effects both from the onset of the masker and from the steady-state portion or offset of the masker. 
TABLE 2. Comparison of parameters of receptive field and MTC for simultaneously recorded single neurons and multiunit clusters

\begin{tabular}{lccccc}
\hline \hline & Single Units & Multiunits & $n$ & $\chi^{2}$ & $P$ \\
\hline Latency, ms & 16.0 & 13.8 & 20 & 9.80 & 0.002 \\
Bandwidth, octaves & 1.5 & 2.24 & 20 & 1.80 & \\
Masker bandwidth, octaves & 2.8 & 2.4 & 13 & 0.69 & \\
Threshold, dB & 42.4 & 40.0 & 20 & 1.25 & \\
Minimum masker level, dB & 39.1 & 40.6 & 12 & 0.08 & \\
Duration, ms & 136.3 & 153.4 & 16 & 1.14 & \\
\hline
\end{tabular}

MTC, masking tuning curve.

\section{IS CUS S I ON}

This study addresses the question of spectrotemporal interaction in the cortical processing of stimulus sequences. Employing a very simple stimulus sequence of two pure tones, it was revealed that the responsiveness of neurons in the auditory cortex depends on the temporal stimulus context. Neural activity can be diminished or enhanced for a period of up to several hundred milliseconds because of the influence of a preceding stimulus. Duration of forward inhibition depended strongly on the frequency and intensity of the first tone of the stimulus pair, raising the question of whether spectral and temporal properties of cortical receptive fields can be described independently. Characteristics of forward inhibition were significantly but weakly linked with various static receptive field properties.

Shapes and sizes of individual MTCs as well as their relation to the frequency response area were generally similar to those reported by Calford and Semple (1995), who studied two-tone interactions with a similar paradigm. Thus the results of the present study provide a confirmation of the findings of Calford et al. The following discussion focuses mainly on new findings, i.e., the time course of forward inhibition, its relation to static receptive field properties, and the comparison of single- and multiunit data. Before we attempt an appropriate interpretation of these results, we first consider some potential effects of the experimentation techniques.

\section{Determination of MTCs}

MTCs were defined as the set of those maskers that inhibited the neural activity of the following probe tone. Inhibition of neural activity was assessed indirectly by presenting a probe tone at various intervals after the masker. If the response strength to the probe was significantly below its response strength if no masker was presented before the probe, a particular masker was inside the MTC. The uninfluenced probe response was estimated by measuring the average probe response strength after the presentation of maskers of very low intensity, under the assumption that a soft masker exerted only a marginal influence on the following neural activity. This value reflected the average probe response strength when probes were presented at the relatively slow repetition rate of the intertrial rate. In the current experiments the intertrial interval was between 350 and 1,030 ms. At these rates, the majority of cortical neurons responded slightly more weakly to the individual stimulus than if stimuli were presented in isolation. Hocherman and Gilat (1981) found that about two-thirds of the neurons in the auditory cortex can have reduced responses if the interstimulus interval is $<1.6 \mathrm{~s}$. Thus our study assessed relative forward inhibition with respect to a habituated state (see also Sutter and Schreiner 1991). Beyond the longest onset interval between masker and probe at which forward inhibition was measured, responses to the probe were still below the value without any preceding sound. The maximal duration of forward inhibition thus represents the longest SOA at which forward inhibition depended on the frequency and intensity of the masker. We did not extend the intertrial interval between the presentation of the stimulus pairs to values $>1.6 \mathrm{~s}$ because of time restrictions. In some cases stimulus interactions for particular SOAs were repeated with a different intertrial interval. In all of these cases MTCs were independent of the intertrial interval.

The major problem in labeling the set of maskers that inhibited the neural activity was that each stimulus pair was presented only once. Similar problems appear when inhibitory subregions of receptive fields are determined ( see also Sutter and Schreiner 1991). The single presentation of randomized tones for the assessment of tuning curves was chosen because it shows little adaptation effect, covers a wide range of tone configurations, and allows post hoc evaluation of the entire response area. The low statistics of the responses to particular tones can be improved by local pooling. This method has the advantage that different aspects of interest can be emphasized, e.g., by averaging only over responses to neighboring frequencies or intensities. In the present investigation, two procedures were applied to arrive at more reliable estimates of MTCs. First, stimulus response areas were low-pass filtered. This procedure was reasonable because most probe response areas could be divided into two main regions. There was one region, usually in the center of the stimulus response area, in which no responses to the probe were present. This central region was surrounded by another region where response strength was not affected by the preceding masker. The transition between the two regions appeared sharp, although we could not determine it accurately. The range of this transition zone was more quantitatively measured by Calford and Semple (1995), who, over a limited frequency and intensity range, presented each masker/probe pair 20 times. Although no values were given, the figures of Calford and Semple indicate that the transition zone was between 0.2 and 0.4 octaves wide. Thus probe response areas basically consisted of two homogenous regions. Within each region, average response strength to particular probes could be estimated by pooling the responses to stimuli that differed only slightly from the stimulus under consideration. After low-pass filtering, those probe responses were labeled as being inhibited where the response strength was below a certain value. Because of the low-pass filtering, we could select a quite liberal criterion for forward inhibition, which was a reduction of the response strength below half the SD of the average response strength of the habituated state. Sizes and shapes of MTCs were quite robust within a reasonable range of criteria and were similar to those derived by visual inspection of probe response areas. The variance of MTCs could be reduced further because forward inhibi- 
tion was measured at different SOAs. This enabled us to compare individual MTC and to discard obvious outliers. In addition, a regression analysis could be performed on the temporal development of various parameters of MTCs, which provided a better estimate of the shape of individual MTCs. Taken together, the quantitative procedures employed in the present study revealed reasonably accurate MTCs. Excitatory subregions of receptive fields were assessed at a higher confidence level than MTCs because stimuli were presented 4-10 times (responses to the masker in the various 2-tone conditions were included for this analysis ).

\section{Effects of anesthesia}

Most experiments were conducted under pentobarbital anesthesia. Pentobarbital is known to decrease the rate of spontaneous activity in the cortex. A reason for this effect may reside in the increase of the inhibitory postsynaptic potentials due to the enhanced sensitivity of $\gamma$-aminobutyric acid-A $\left(\mathrm{GABA}_{\mathrm{A}}\right)$ receptors (Franks and Lieb 1994). Therefore the temporal response characteristics of cortical cells obtained in this study have to be considered carefully. A way to estimate the potential influence of the anesthesia is to compare the temporal dynamics obtained with different anesthetic drugs. Two of our experiments (10 neurons) were performed with the use of ketamine (supplemented by azepromacine). Between the two types of anesthesia no obvious difference in any of the spectrotemporal characteristics of forward inhibition was observed. Similar results were reported by Calford and Semple (1995), who studied twotone interaction with the same drugs. Thus the results of the present study are consistent between the anesthetics used throughout the experiment. Recordings of neural discharges from awake guinea pigs (Creutzfeldt et al. 1980) and recordings of intracortical slow-wave activity in cats (Etholm et al. 1976) have shown that, in awake preparations, forward inhibition is also present, although time constants may be shorter and strength of inhibition may be weaker. To what extent the results of the present study on other properties of forward inhibition apply to unanesthetized animals, however, still remains to be determined.

\section{Single-neuron versus multiple-neuron activity}

The interpretation of the present study has to consider that recordings were made from both single neurons and from multiunit clusters. Multiunit activity reflects the action potentials generated by several neurons in the vicinity of the electrode tip. Because large cells have action potentials with greater amplitudes, extracellular single-unit recordings are most likely exclusively made from pyramidal cells, whereas other cell types such as stellate cells also contribute to multiunit recordings.

There were several reasons for including multiunit clusters in our study. The tests for the spectrotemporal characteristics of forward inhibition required a time period of $\geq 1 \mathrm{~h}$. Singleunit recordings frequently could not be maintained over such long periods. Therefore recordings of multiunit activity provided a way to study forward inhibition in more detail and to record from more cortical locations so that the data base was increased.
Recordings of single-unit and multiunit neuron activity need not necessarily reveal similar response characteristics. Generally, the two to five MTCs that are probed with the multiunit activity can be related to each other in three ways. MTCs of individual neurons could be nonoverlapping. In this case, the resulting probe response area of the cluster would consist of several regions within which the average response strength is constant. The size of the cluster MTC depends on how the statistics of the probe response areas of the individual neurons are related to each other, such that MTCs of the cluster could be as small as an MTC of a single neuron, or could be the sum of the MTCs of all single neurons in the cluster. If this were the case, variability of MTCs from simultaneously recorded single- and multiunit activity should be very high, which was not observed among the recording sites of the present study. In addition, probe response areas of a cluster would not consist of regions where no probe responses are observed. This is also in contrast to the cluster MTCs found in the present experiments. The second possibility is that MTCs of individual neurons of a cluster are partially overlapping. Depending on the degree of overlap of individual MTCs, probe response areas of clusters would then be made up of individual regions with different average response strengths. Thus there would be extended transition zones within the MTC between areas with no probe responses and regions where probe tones are not affected by the preceding masker. This is also not in accordance with the findings of the present study. The third possibility is that MTCs of individual neurons are identical. This option is mostly in agreement with the results of the present study. In cases in which single- and multiunit activity was recorded in parallel, temporal properties of forward inhibition of the two groups were not significantly different from each other (Table 2). The same held true when the total sample of single- and multiunit recordings was compared. Our results thus indicate that adjacent neurons in the auditory cortex process temporal information in a similar manner. Therefore results of single- and multiunit recordings with regard to forward inhibition will be discussed conjointly. The argumentation is different when excitatory receptive fields of single neurons and clusters are compared. If excitatory receptive fields of the individual neurons of a cluster have the same extent, no difference between single-neuron and cluster receptive fields will be found. If receptive fields of the individual neurons of a cluster are partially overlapping or nonoverlapping, the receptive field of the cluster will be the sum of the individual receptive fields. The latter possibility is in accordance with the results of the present study because bandwidths of single neurons were significantly smaller than bandwidths of clusters (for discussion see also Schreiner and Mendelson 1990).

\section{Generation of forward inhibition}

Several mechanisms have to be considered regarding where and how forward inhibition/suppression of cortical neurons is generated. Nonneural causes such as suppressive effects of nonlinearities in basilar membrane motion, noninhibitory causes such as adaptation, and neural inhibitory effects accumulate and combine along the auditory pathway until they are revealed in the cortex by the forward masking 
technique applied in this study. Because most conditions in this study did not involve simultaneous presentation of masker and probe, instantaneous cochlear mechanic suppression effects did not contribute significantly to the observed effects.

Another possible source of forward suppression is neuronal fatigue or adaptation. In the auditory nerve, the amount of forward suppression is proportional to the excitation induced by the previous masker (Harris and Dallos 1979; Smith 1977). Consequently it is seen only for stimuli within the excitatory receptive field of the neuron. This and previous studies (Calford and Semple 1995; Phillips et al. 1989) have found that a significant number of neurons was inhibited from stimuli outside their excitatory receptive field, indicating that adaptation effects from subcortical stages of the auditory pathway, including the auditory nerve, cannot be the sole sources of the observed effects. Forward inhibition of the activity of cortical cells may reflect inhibitory properties already present at subcortical stages of the auditory pathway as well as cortical influences. Numerous studies have demonstrated that forward inhibition of neural responses is present on all levels of the auditory system, beginning with the cochlear nuclear complex (Boettcher et al. 1990; Kaltenbach et al. 1993; Shore 1995), up to the medial geniculate body (Schreiner 1981). At successive stages of the auditory pathway, new properties of forward inhibition emerge. In the cochlear nuclear complex, $\sim 20 \%$ of the neurons are inhibited from stimuli outside the excitatory subfields of their receptive field. In addition, there are neurons with nonmonotonous time courses of forward inhibition (Kaltenbach et al. 1993; Shore 1995). For these neurons, suppression of the probe response was not present until several tens of milliseconds after the masker offset. In the medial geniculate body there is evidence that neurons can also be inhibited from stimuli outside the receptive field (Schreiner 1981). Thus, with regard to the observed stimulus range of forward inhibition, subcortical neurons could already account for the properties of cortical neurons.

An important temporal characteristic of forward inhibition is its duration. In the auditory nerve of the Mongolian gerbil, recovery from forward inhibition was in the range between 40 and $310 \mathrm{~ms}$, with an average of $115 \mathrm{~ms}$ (Smith 1977). Similar values were also found for the chinchilla (Harris and Dallos 1979). For the anteroventral cochlear nucleus, Shore (1995) reported slightly longer recovery times than in the auditory nerve. In the medial geniculate body of awake guinea pigs, the recovery time constants were found to be up to twice the time constants of those found in the auditory nerve (Schreiner 1981). In our study the maximal duration of forward inhibition was in the range of 40 and $430 \mathrm{~ms}$, with a mean of $143 \mathrm{~ms}$, and thus not much longer than in subcortical stages of the auditory pathway. Different time constants, however, have to be considered carefully. First, in all neural structures that have been investigated so far, forward inhibition has been studied with maskers of different intensity, frequency, and duration. Second, studies have been performed on different species and under different anesthesia. Therefore, with regard to the duration of forward inhibition, no conclusion can be drawn as to the origin of cortically revealed forward inhibition.
Duration of forward inhibition can also be estimated from studies on time-varying stimuli such as amplitude- and frequency-modulated sounds. Comparison of modulation transfer functions at various stages of the auditory system shows that both high-frequency cutoffs of modulation transfer functions and the best modulation frequencies shift progressively downward along the auditory pathway (reviewed in Langner 1992). Neurons in the auditory nerve can phase lock their responses to modulation rates $>1,000 \mathrm{~Hz}$ (Joris and Yin 1992), whereas in cortical auditory fields, most neurons have cutoff frequencies of $\sim 10 \mathrm{~Hz}$ (Creutzfeldt et al. 1980; Eggermont 1994; Schreiner and Urbas 1986, 1988). Thus studies with amplitude- and frequency-modulated sounds indicate that cortical mechanisms contribute to the generation of temporal response properties of cortical neurons.

A main difference between subcortical and cortical neurons, however, appears to be the strength of inhibition. Whereas subcortical neurons, including those in the medial geniculate body (Schreiner 1981), exhibit only a gradual suppression of neural discharges, most cortical neurons are completely inhibited after a masker stimulus. Thus, in conclusion, there is evidence that the forward inhibition of cortical neurons is generated, in part, by intracortical mechanisms ( see also Calford and Semple 1995). This view is further supported by a study of visual forward masking that compared temporal stimulus interactions in the lateral geniculate body and the visual cortex (Nelson 1991a,b).

Adaptation of cortical cells can, in part, account for the reduction of neural responsiveness observed after the presentation of the masker. This was suggested by Phillips and coworkers (Phillips 1985, 1990; Phillips and Cynader 1985; Phillips and Sark 1991), who found with simultaneous tone interactions that reduction of responsiveness and prolongation of response latencies are due to adaptation (e.g., Fig. $6)$, whereas no significant changes of latencies are seen when a cell receives competing excitatory and inhibitory inputs. Adaptation, however, cannot account for all the forward inhibition effects observed in cortical cells, because it was also induced from maskers to which the neuron under consideration did not respond. Thus inhibitory postsynaptic potentials also have to be involved in the cortical forward inhibition.

For most cortical neurons, forward inhibition is chiefly induced from stimuli within the excitatory subregions of the receptive field. Furthermore, forward inhibition usually lasts the longest for a $\mathrm{CF}$ tone. This indicates that temporal interactions are mainly among neurons with overlapping receptive fields. Thus forward inhibition emerges probably mainly from neural interactions within the same isofrequency domain, whereas neurons of different isofrequency domains have a smaller contribution. The type of neural interactions is still unknown, but may be similar to those operating in the visual cortex. There, application of bicuculline resulted in no change of the duration and type of temporal stimulus interactions. This indicates that forward inhibition is not induced by $\mathrm{GABA}_{\mathrm{A}}$-mediated inhibitory postsynaptic potentials (Nelson 1991b). This view is further supported by our finding that there was no obvious difference in the properties of forward inhibition between the neurons that were investigated under barbiturate and ketamine anesthesia, although barbiturates are known to potentiate the ef- 
fect of $\mathrm{GABA}_{\mathrm{A}}$ receptors (Franks and Lieb 1994). Results thus are indicative of presynaptic inhibition, possibly at the geniculate-cortical synapse, or of shunting inhibition at the cell soma. This temporal inhibition possibly provides a mechanism similar to "spatial" lateral inhibition. Whereas this type of inhibition suppresses the activity of adjacent neurons and thus enhances the spatial contrast, the former suppresses neural activity of successive time intervals and thus sharpens the temporal contrast.

\section{Comparison with previous studies: inhibitory neural interactions}

The capability of cortical neurons to process temporal aspects of stimuli has usually been assessed with doublepulse or periodically modulated stimuli. The latter have revealed modulation transfer functions that describe how a cell responds to different types of periodically modulated sounds, such as amplitude-modulated pure tones or noise, or click trains. Rate modulation transfer functions are related to the present results of forward masking, because both show how the discharge rate of neurons depends on the temporal interval between two acoustic transients. The comparison of the results obtained with the two paradigms, however, has to be taken into consideration as follows.

Modulation transfer functions have commonly been assessed with a constant carrier (usually a pure tone or noise) whose envelope is periodically modulated. In the present study, the stimulus was a long sequence of elements differing in frequency and intensity. In addition there were two temporal intervals between successive elements. The modulation alternated between a shorter interval between the masker and probe, which was in the range of 30 and $430 \mathrm{~ms}$, and a longer interval between the probe and the following masker, which was between 290 and $540 \mathrm{~ms}$. With this paradigm it was found that the strength of probe responses, on average, was specifically inhibited up to an SOA of $143 \mathrm{~ms}$. The range of forward inhibition was quite wide and between 40 and $430 \mathrm{~ms}$. The average value for forward inhibition is similar to the values reported for modulation transfer functions. Variation of the modulation rate has shown that neurons in auditory cortex encode only slowly modulated sounds. Transfer functions were of the low-pass or band-pass type with limiting rates between 4 and $28 \mathrm{~Hz}$ (Creutzfeldt et al. 1980; Eggermont 1991, 1994; Müller-Preuß 1986; Phillips et al. 1989; Schreiner and Urbas 1986, 1988; but see deRibaupierre et al. 1972). The present study shows that maximal duration of forward inhibition, however, depended strongly on the spectral composition of consecutive elements. Forward inhibition lasted the longest if successive tones had the same frequency and were at the $\mathrm{CF}$ of the neuron. Duration of forward inhibition, however, was significantly shorter if successive elements differed in frequency, even if they were still clearly inside the excitatory receptive field. Because modulation transfer functions have mostly been determined with CF tones, they only describe the lower limits to which cortical neurons can encode modulated sounds. For sounds in which not only does the envelope vary over time but also the spectral composition changes in time, results of the present study indicate that neurons can encode sounds with modulation rates above those predicted by the cutoff frequency of the traditional modulation transfer function.

The dependence of neural responses on the time context has been addressed previously. In three independent studies, examples were reported that showed that the neural responsiveness depended on the spectral content of the preceding stimulus. In a study on awake cats, McKenna and colleagues (1989) found that $84 \%$ of neurons in the auditory cortex responded differently to a tone presented alone compared with the same tone when presented within a sequence of different tones. Both inhibition and facilitation of responses was observed. In auditory cortex of anesthetized monkeys, combination selective cells were found. These cells responded vigorously to a particular tone only if it was preceded by another tone at a specific frequency and at a specific temporal interval (Riquimaroux 1994). Recently, studying nonsimultaneous two-tone interactions in AI of anesthetized cats, Calford and Semple (1995) reported of several neurons for which MTCs decreased in size as the masker-to-probeonset interval increased, similar to the present findings.

Another property that affects the temporal response characteristics is the intensity of the stimulus. Phillips et al. (1989) reported that numerous neurons entrain their discharges to higher modulations the higher the total intensity of the stimulus. This behavior was mostly found for neurons with monotonic rate-intensity functions. For neurons with nonmonotonic rate-intensity functions, however, increasing the stimulus intensity could result in a decrease of the modulation rate to which a neuron responded. These findings were confirmed and extended by Calford and Semple (1995) and the present study: with sequences of tones of different intensity, the longest-lasting forward inhibition was not always induced from the most intense maskers. For a significant number of neurons, maskers at intermediate intensities induced longer periods of inhibition than loud maskers. Calford and Semple concluded that the sources for inhibition responsible for nonmonotonic rate-intensity functions and forward inhibition (at least for long SOAs) are not necessarily identical.

Stimulus interactions do not only depend on properties of the carrier but also on envelope properties. Here we found that the duration of the individual elements of a sequence strongly affects the duration of forward inhibition (see also Calford and Semple 1995). Long maskers induced longer periods of forward inhibition than did short maskers. That means that neurons respond with higher discharge rates to a sound that is modulated at a particular repetition rate if duration of the elements of the sequence is short and pauses are long than if stimulus duration is long and pauses are short. Another dependence on the modulation envelope has already been uncovered with periodically modulated sounds. It was found that best modulation frequencies are the higher the sharper are the transients of the modulator (Eggermont 1994; Schreiner and Urbas 1988). Influence of slope characteristics of the masker and probe were not investigated in the present study, but these probably also affected the duration of forward inhibition.

\section{Comparison with previous studies: latency}

The previous paragraphs discuss how the temporal stimulus context affects the discharge rate of cortical neurons. In 
addition to forward inhibition, the temporal stimulus context can also affect the latent period of neural discharges. Phillips et al. (1989), using trains of tone bursts, found that the latency of the first spike response to each tone increased when the temporal interval between the tones was decreased. Similar results were reported for sequences of tone bursts of different frequency and intensity (Calford and Semple 1995; present study) and for click trains (Eggermont 1991). In all of these studies latency increases were associated with a decreased neural discharge probability. In addition to previous reports on temporal stimulus interactions, the present study found cases for which the latencies of neural responses were lengthened without concomitant alterations of the response strength (e.g., Fig. 4). This indicates that rate and latency of neural discharges can vary independently of each other. Thus discharge rate and timing of discharges could also provide independent neural mechanisms for the encoding of time-varying sounds.

\section{Relation of spectral and temporal properties of receptive fields}

The previous discussion shows that neural responses to a sound depend on the temporal context within which the sound occurs. This implies that the set of stimuli that affects neural activity is not only dependent on the spectral composition of sounds but also on the temporal evolution of sounds. Receptive fields of auditory neurons thus extend both in the spectral and temporal domain. These two domains are not necessarily separable. A specific relation between the spectral and temporal aspects of the response characteristics of auditory neurons has been found for various cell types in the cochlear nucleus (reviewed in Langner 1992). A combined approach provides the concept of the spectrotemporal receptive field, which is derived from a calculation of a linear function of the second-order Volterra kernel for a special class of stimuli (Aertsen and Johannesma 1980). For cortical neurons the relation between the spectral and temporal aspects of receptive fields has, with a few exceptions, not yet been established.

The analysis of the present study reveals a weak but specific relationship between the spatial and temporal dimension of receptive fields of cortical neurons. First, duration of temporal stimulus interactions depended on the CF of the neurons. Neurons with low CFs were inhibited for longer periods than neurons with high CFs. This relation between $\mathrm{CF}$ and forward inhibition is interesting because it parallels findings of perceptual forward masking where the duration of forward masking decreased with the frequency of the stimuli (Jesteadt et al. 1982). Because in subcortical stages of the auditory pathway duration of forward inhibition is independent of the neuron's CF, results suggest that cortical neurons may be involved in generating perceptual forward masking effects.

Duration of forward inhibition varied also with other properties of cortical cells, such as bandwidth and latency of neural responses. Neurons with small receptive fields and short latencies tended to have shorter periods of forward inhibition compared with neurons with larger receptive fields and long latencies. Although these correlations were weak, values were comparable with those found between different static receptive field parameters (Schreiner and Mendelson 1990; Schreiner et al. 1992). Our findings therefore could indicate that there are functionally specialized neurons in AI that have a high spectral and temporal resolution and that respond shortly after the presentation of a stimulus, and that there are other neurons that integrate stimuli over a wider spectral and temporal range and have long latency periods. A similar relationship has also been observed in a cortical study of repetition coding of acoustic and electrical cochlear stimulation in which best following rate and onset latency were inversely correlated (Schreiner and Raggio 1996). Further evidence comes from recordings from the auditory midbrain in which the latency of neural responses was negatively correlated with the preferred modulation rate (Langner et al. 1987). In conclusion, these findings suggest that the association between the latency of neural responses and the duration of temporal stimulus interactions provides a facilitation for the processing of time-varying sounds.

It is well established that static receptive field properties, such as receptive field bandwidth, response threshold, and others, are distributed in an orderly fashion along isofrequency contours of AI (Heil et al. 1992, 1994; Rajan et al. 1990; Schreiner and Mendelson 1990; Schreiner et al. 1992). In the present study we found that the bandwidth was weakly correlated with several temporal aspects of response characteristics of cortical neurons, including maximal duration of forward inhibition and temporal course of the minimal inhibitory masker intensity and of inhibitory frequency range (Table 1). Results of the present study thus give rise to the speculation that temporal response characteristics of neurons are also topographically organized within AI. This hypothesis is further supported by an investigation of some of the authors who studied stimulus interactions with sequences of five tone bursts with different temporal intervals between the elements of the sequence (Krüger and Schreiner 1994). It was found that neurons in the ventral third of the isofrequency domain had shortest recovery periods, whereas recovery was longer for neurons that were located more ventrally and dorsally.

\section{Concluding remarks}

Three concepts have traditionally been proposed for the neural encoding of acoustic information in the auditory system: the rate code, the temporal code, and the place code. In the present study we found that neural responses to the same stimulus were different if the stimulus occurred within a sequence of other stimuli compared with being presented in isolation. Thus the present study provides evidence that information about stimulus sequences may be coded in the auditory cortex by changes in the discharge rate (response strengths both decreased and increased), and by changes in the timing of neural responses. In addition, there were indications that neurons with different temporal response characteristics are located at different regions of AI.

The dependence of forward masking on the stimulus shows many similarities to the characteristics of perceptual temporal stimulus interaction. For example, duration of psychophysical forward masking acts in a temporal range similar to the range of cortical forward inhibition, and it also depends on the frequency similarity of masker and probe 
(Jesteadt et al. 1982). Similar dependencies have been described for rhythmic grouping of tone sequences and stream segregation and integration of sequences (reviewed in Bregman 1990). How these changes of the neural activity in the cortex are involved in the generation of these perceptions and their role in the analysis of complex auditory scenes, however, still remains an open question.

We thank Dr. Barbara Calhoun and S. Wong for help during some of the experiments.

This work was supported by the Office of Naval Research (N00014-94-10547) National Institute of Deafness and Other Communications Disorders Grant DC-22260, and Deutsche Forschungsgemeinschaft (Br 1385/1).

Present address and address for reprint requests: M. Brosch, Institut für Neurobiologie, Brenneckestraße 6, 39118 Magdeburg, Germany.

Received 28 December 1995; accepted in final form 24 October 1996.

\section{REFERENCES}

Aertsen, A. M. H. J. And Johannesma, P. I. M. Spectro-temporal receptive fields of auditory neurons in the grassfrog. Biol. Cybern. 38: 223-234, 1980.

Boettcher, F. A., Salvi, R. J., AND Saunders, S. S. Recovery from shortterm adaptation in single neurons in the cochlear nucleus. Hear. Res. 48: 125-144, 1990.

Bregman, A. S. Auditory Scene Analysis. The Perceptual Organization of Sound. Cambridge, MA: MIT Press, 1990.

Brosch, M. AND Schreiner, C. E. Suppressive and facilitatory effects of stimulus sequences on neural responses in cat primary auditory cortex. Soc. Neurosci. Abstr. 20: 141.16, 1994.

Calford, M. B. and Semple, M. N. Monaural inhibition in cat auditory cortex. J. Neurophysiol. 73: 1876-1891, 1995.

CreutzFeldt, O. D., Hellweg, F. C., And Schreiner, C. E. Thalamocortical transformation of responses to complex auditory stimuli. Exp. Brain Res. 39: 87-104, 1980.

De Ribaupierre, F., Goldstein, M. H., and Yeni-Komshian, G. Cortical coding of repetitive acoustic pulses. Brain Res. 48: 205-225, 1972.

Diamond, I. T., Goldberg, J. M., And NeFF, W. D. Tonal discrimination after ablation of the auditory cortex. J. Neurophysiol. 25: $223-$ $235,1962$.

DiAmond, I. T. AND NefF, W. D. Ablation of temporal cortex and discrimination of auditory patterns. J. Neurophysiol. 20: 300-315, 1957.

EGGERMONT, J. J. Rate and synchronization measures for periodicity coding in primary auditory cortex. Hear. Res. 56: 153-167, 1991.

EGGERMONT, J. J. Temporal modulation transfer functions for AM and FM stimuli on cat auditory cortex. Effects of carrier type, modulating waveform and intensity. Hear. Res. 74: 51-66, 1994.

Etholm, B., Guerstad, L. I., And Skrede, K. K. Size and duration of inhibition in the medial geniculate body of unanesthetized cats. Acta Otolaryngol. 81: 102-112, 1976.

FRANKS, N. P. AND LIEB, W. R. Molecular and cellular mechanisms of general anaesthesia. Nature Lond. 367: 607-613, 1994.

Glass, I. AND Wollberg, Z. Auditory cortex responses to sequences of normal and reversed squirrel monkey vocalizations. Brain Behav. Evol. 22: $13-21,1983$.

HaRris, D. M. AND Dallos, P. Forward masking of auditory nerve fiber responses. J. Neurophysiol. 42: 1083-1107, 1979.

HefFner, H. E. And HefFner, R. S. Temporal lobe lesions and perception of species-specific vocalizations by macaques. Science Wash. DC 226 75-76, 1984.

HefFNER, H. E. AND HeFFNER, R. S. Effect of restricted cortical lesions on absolute threshold and aphasia-like deficits in Japanese macaques. Behav. Neurosci. 103: 158-169, 1989.

Heil, P., Rajan, R., and Irvine, D. R. F. Sensitivity of neurons in cat primary auditory cortex to tones and frequency modulated stimuli. II. Topographic representation along the "isofrequency" dimension. Hear. Res. 63: 135-156, 1992.

Heil, P., Rajan, R., and IRvine, D. R. F. Topographic representation of tone intensity along the isofrequency axis of cat primary auditory cortex. Hear. Res. 76: 188-202, 1994.
HOCHERMAN, S. AND GILAT, E. Dependence of auditory evoked unit activity on interstimulus interval in the cat. J. Neurophysiol. 45: 987-997, 1981.

HuPfER, K., JÜrgens, U., AND PloOG, D. The effect of superior temporal lesions on the recognition of species-specific calls in the squirrel monkey. Exp. Brain Res. 30: 75-87, 1977.

Jesteadt, W., Bacon, S. P., And Lehman, J. R. Forward masking as a function of frequency, masker level and signal delay. J. Acoust. Soc. Am. 71: 950-962, 1982.

JoRIS, P. X. AND YIN, T. C. T. Responses to amplitude-modulated tones in the auditory nerve of the cat. J. Acoust. Soc. Am. 91: 215-232, 1992.

KaAs, J. H., Axelrof, S., And Diamond, I. T. An ablation study of the auditory cortex in the cat using binaural tonal patterns. J. Neurophysiol. 30: 710-724, 1967.

Kaltenbach, J. A., Meleca, R. J., Falzarano, P. R., Myers, S. F., and Simpson, T. H. Forward masking properties of neurons in the dorsal cochlear nucleus: possible role in the process of echo suppression. Hear. Res. 67: 35-44, 1993.

KrÜ Ger, K. AND Schreiner, C. E. Processing of tone sequences in the cat primary auditory cortex. Soc. Neurosci. Abstr. 20: 141.7, 1994.

LANGNER, G. Periodicity coding in the auditory system. Hear. Res. 60: $115-142,1992$.

Langner, G., Schreiner, C. E., and Merzenich, M. M. Covariation of latency and temporal resoluation in the inferior colliculus of the cat. Hear. Res. 31: 197-202, 1987.

LÜ SCHER, E. AND ZWISLOCKI, J. The decay of sensation and the remainder of adaptation after short pure tone impulses on the ear. Acta Otolaryngol. 35: 428-446, 1947.

McKenna, T. M., Weinberger, N. M., And Diamond, D. M. Responses of single auditory cortical neurons to tone sequences. Brain Res. 481: $142-153,1989$.

Múller-Preuß, P. On the mechanisms of call coding through auditory neurons in the squirrel monkey. Eur. Arch. Psychiatry Neurol. Sci. 236: $50-55,1986$.

Nelson, S. B. Temporal interactions in the cat visual system. Orientationselective suppression in the visual cortex. J. Neurosci. 11: 344-356, 1991a.

Nelson, S. B. Temporal interactions in the cat visual system. Pharmacological studies of cortical suppression suggest a presynaptic mechanism. $J$. Neurosci. 11: 369-380, 1991b.

Newman, J. D. Primate hearing mechanisms. In: Comparative Primate Biology, edited by H. D. Steklis and J. Erwin. New York: Liss, 1988, p. 469499.

PhILLIPS, D. P. Stimulus intensity and loudness recruitment: neural correlates. Hear. Res. 19: 253-268, 1985.

Phillips, D. P. Neural representation of sound amplitude in the auditory cortex: effects of noise masking. Behav. Brain Res. 37: 197-214, 1990.

Phillips, D. P. AND Cynader, M. S. Some neural mechanisms in the cat's auditory cortex underlying sensitivity to combined tone and wide spectrum noise stimuli. Hear. Res. 18: 87-102, 1985.

Phillips, D. P., Hall, S. E., AND Hollett, J. L. Repetition rate and signal level effects on neuronal responses to brief tone pulses in cat auditory cortex. J. Acoust. Soc. Am. 85: 2537-2549, 1989.

PhILLIPS, D. P. AND SARK, S. A. Separate mechanisms control spike number and inter-spike intervals in transient responses of cat auditory cortex neurons. Hear. Res. 53: 17-27, 1991.

Rajan, R., Aitkin, L. M., and Irvine, D. R. F. Azimuthal sensitivity of neurons in primary auditory cortex of cats. II Organization along frequency-band strips. J. Neurophysiol. 64: 888-902, 1990.

Riquimaroux, H. Neuronal scene analysis? Basic evidence found in the monkey primary auditory cortex. Trans. Tech. Committee Psychol. Physiol. Acoustics Acoust. Soc. Jpn. 29: 1-8, 1994.

Scharlock, D. P., NefF, W. D., and Strominger, N. L. Discrimination of tone duration after bilateral ablation of cortical auditory areas. $J$. Neurophysiol. 28: 673-681, 1965.

SCHREINER, C. E. Poststimulatory effects in the medial geniculate body of guinea pigs. In: Neuronal Mechanisms of Hearing, edited by J. Syka and L. Aitkin. New York: Plenum, 1981, p. 191-196.

Schreiner, C. E. AND Langner, G. Coding of temporal patterns in the central auditory system. In: Auditory function, edited by G. M. Edelman, W. E. Gall, and W. M. Cowan. New York: Wiley, 1988, p. 337-361.

SCHREINER, C. E. AND Mendelson, J. R. Functional topography of cat pri- 
mary auditory cortex: distribution of integrated excitation. J. Neurophysiol. 64: 1442-1459, 1990

Schreiner, C. E., Mendelson, J. R., And Sutter, M. L. Functional topography of cat primary auditory cortex: representation of tone intensity. Exp. Brain Res. 92: 105-122, 1992.

Schreiner, C. E. AND RAGGio, M. W. Neuronal responses in cat auditory cortex to electrical cochlear stimulation. II. Repetition coding. J. Neurophysiol. 75: 1283-1300, 1996.

SCHREINER, C. E. AND URBAS, J. V. Representation of amplitude modulation in the auditory cortex of the cat. I. The anterior auditory field (AAF). Hear. Res. 21: 227-241, 1986.

SCHREINER, C. E. AND URBAS, J. V. Representation of amplitude modulation in the auditory cortex of the cat. I. Comparison between cortical fields. Hear. Res. 32: 49-64, 1988.

SHORE, S. E. Recovery of forward-masked responses in ventral cochlear nucleus neurons. Hear. Res. 82: 31-43, 1995.

SMITH, R. L. Short-term adaptation in single auditory nerve fibers: some poststimulatory effects. J. Neurophysiol. 40: 1098-1112, 1977.

Steinschneider, M., Schroeder, C. E., Arezzo, J. C., And Vaughan, H. G., JR. Speech-evoked activity in primary auditory cortex: effects of voice onset time. Electroencephalogr. Clin. Neurophysiol. 92: 30-43, 1994.

Stevens, S. S. and Davis, H. Hearing. Its Psychology and Physiology. New York: Wiley, 1938.

Strominger, N. L., Oesterreich, R. E., And NefF, W. D. Sequential auditory and vsiual discriminations after temporal lobe ablation in monkeys. Physiol. Behav. 24: 1149-1156, 1980.

SuTter, M. L. AND SCHREINER, C. E. Physiology and topography of neurons with multipeaked tuning curves in cat primary auditory cortex. J. Neurophysiol. 65: 1207-1226, 1991.

WANG, X., Merzenich, M. M., Beitel, R., And Schreiner, C. E. Representation of a species-specific vocalization in the primary auditory cortex of the common marmoset: temporal and spectral characteristics. J. Neurophysiol. 74: 2685-2706, 1995. 